\title{
The initial conditions of star formation in the Ophiuchus main cloud: Kinematics of the protocluster condensations ${ }^{\star}, \star \star$
}

\author{
Ph. André ${ }^{1,2}$, A. Belloche ${ }^{1,3}$, F. Motte ${ }^{1,2}$, and N. Peretto ${ }^{1,4}$ \\ ${ }^{1}$ CEA/DSM/DAPNIA, Service d'Astrophysique, CE Saclay, Orme des Merisiers, 91191 Gif-sur-Yvette, France \\ e-mail: pandre@cea.fr \\ 2 Laboratoire AIM, Unité Mixte de Recherche CEA/DSM - CNRS - Université Paris Diderot, CE Saclay, France \\ 3 Max-Planck-Institut für Radioastronomie, Auf dem Hügel 69, 53121 Bonn, Germany \\ 4 Physics \& Astronomy Department, University of Manchester, PO Box 88, Manchester M60 1QD, UK
}

Received 6 March 2007 / Accepted 7 June 2007

\section{ABSTRACT}

\begin{abstract}
Context. The earliest phases of clustered star formation and the origin of the stellar initial mass function (IMF) are currently much debated. In one school of thought the IMF of embedded clusters is entirely determined by turbulent fragmentation at the prestellar stage of star formation, while in a major alternative view it results from dynamical interactions and competitive accretion at the protostellar stage.

Aims. In an effort to discriminate between these two pictures for the origin of the IMF, we investigated the internal and relative motions of starless condensations and protostars previously detected by us in the dust continuum at $1.2 \mathrm{~mm}$ in the L1688 protocluster of the Ophiuchus molecular cloud complex. The starless condensations have a mass spectrum resembling the IMF and are therefore likely representative of the initial stages of star formation in the protocluster.

Methods. We carried out detailed molecular line observations, including some $\mathrm{N}_{2} \mathrm{H}^{+}(1-0)$ mapping, of the Ophiuchus protocluster condensations using the IRAM $30 \mathrm{~m}$ telescope.

Results. We measured subsonic or at most transonic levels of internal turbulence within the condensations, implying virial masses which generally agree within a factor of $\sim 2$ with the masses derived from the $1.2 \mathrm{~mm}$ dust continuum. This supports the notion that most of the L1688 starless condensations are gravitationally bound and prestellar in nature. We detected the classical spectroscopic signature of infall motions in $\mathrm{CS}(2-1), \mathrm{CS}(3-2), \mathrm{H}_{2} \mathrm{CO}\left(2_{12}-1_{11}\right)$, and/or $\mathrm{HCO}^{+}(3-2)$ toward six condensations, and obtained tentative infall signatures toward 10 other condensations. In addition, we measured a global one-dimensional velocity dispersion of less than $0.4 \mathrm{~km} \mathrm{~s}^{-1}$ (or twice the sound speed) between condensations. The small relative velocity dispersion implies that, in general, the condensations do not have time to interact with one another before evolving into pre-main sequence objects.

Conclusions. Our observations support the view that the IMF is partly determined by cloud fragmentation at the prestellar stage. Competitive accretion is unlikely to be the dominant mechanism at the protostellar stage in the Ophiuchus protocluster, but it may possibly govern the growth of starless, self-gravitating condensations initially produced by gravoturbulent fragmentation toward an IMF, Salpeter-like mass spectrum.
\end{abstract}

Key words. stars: formation - stars: circumstellar matter - ISM: clouds - ISM: structure - ISM : kinematics and dynamics ISM: molecules

\section{Introduction}

While most stars are believed to form in clusters (e.g. Adams \& Myers 2001; Lada \& Lada 2003), our present theoretical understanding of the star formation process is essentially limited to isolated dense cores and protostars (e.g. Shu et al. 1987, 2004). Studying the formation and detailed properties of prestellar condensations in cluster-forming clouds is thus of prime importance if we are to explain the origin of the stellar initial mass function (IMF). Some progress has recently been made in this area (cf.

* Based on observations carried out with the IRAM 30-m telescope. IRAM is supported by INSU/CNRS (France), MPG (Germany), and IGN (Spain).

$\star \star \mathrm{N}_{2} \mathrm{H}^{+}(1-0)$ data cubes in FITS format are available at the CDS via anonymous ftp to cdsarc.u-strasbg.fr (130.79.128.5) or via http://cdsweb.u-strasbg.fr/cgi-bin/qcat?J/A+A/472/519
André et al. 2000; Motte \& André 2001; Ward-Thompson et al. 2007 for reviews).

On the observational side, recent ground-based (sub)millimeter continuum surveys of a few nearby cluster-forming clouds such as the L1688 clump in Ophiuchus (also known as the $\rho$ Ophiuchi main cloud; $d \sim 150 \mathrm{pc}$ ), the Serpens central clump ( $d \sim 300$ pc), or the NGC 2068/2071 clumps in Orion B $(d \sim 400 \mathrm{pc})$ have uncovered "complete" samples of prestellar condensations whose associated mass distributions resemble the stellar IMF (e.g. Motte et al. 1998 - MAN98; Testi \& Sargent 1998; Johnstone et al. 2000; Motte et al. 2001; Bontemps et al. 2001; and references therein). In particular, this is the case for the population of 57 starless condensations identified by MAN98 in their $1.2 \mathrm{~mm}$ continuum mosaic of L1688 with the MPIfR bolometer array (MAMBO) on the IRAM $30 \mathrm{~m}$ telescope. These Ophiuchus condensations, which were identified using a multi-resolution analysis equivalent to a wavelet decomposition 


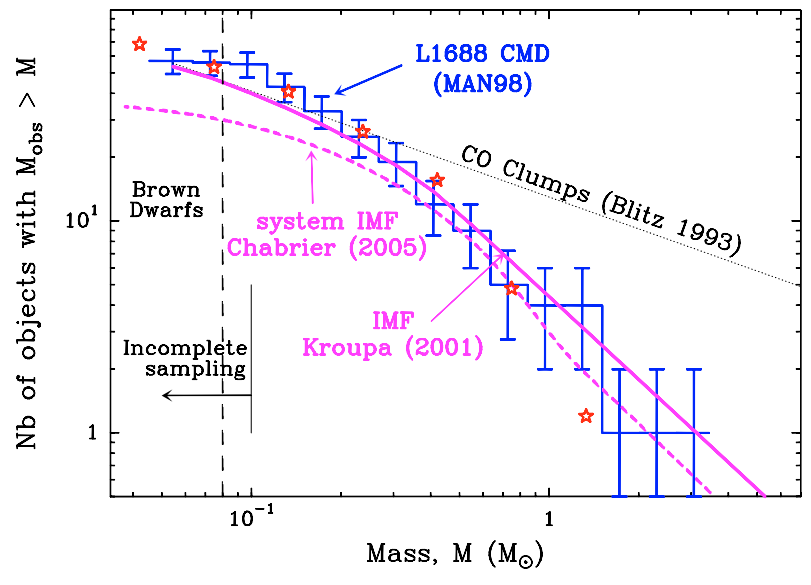

Fig. 1. Cumulative mass distribution of the 57 starless condensations identified by MAN98 in the L1688 protocluster (histogram with error bars). For comparison, the solid curve shows the shape of the field star IMF (e.g. Kroupa 2001), while the dashed curve corresponds to the IMF of multiple systems (e.g. Chabrier 2003, 2005). The star markers represent the mass function of (primary) pre-main sequence objects in L1688 as derived from the ISOCAM mid-IR survey of Bontemps et al. (2001). The dotted line shows a $N(>M) \propto M^{-0.6}$ power-law distribution corresponding to the typical mass spectrum found for CO clumps (see Blitz 1993; Kramer et al. 1998). Note the flattening of the mass distributions below $\sim 0.4 M_{\odot}$ and the apparent excess of starless condensations over stellar systems at the low-mass end.

(cf. Starck et al. 1998; Motte et al. 2003), are seen on the same spatial scales as protostellar envelopes (i.e., 2300-4500 AU or $\sim 15^{\prime \prime}-30^{\prime \prime}$ in L1688). Their mass spectrum is consistent with the Salpeter (1955) power-law IMF at the high-mass end and shows a tentative break at $\sim 0.4 M_{\odot}$ (see Fig. 1). This break is reminiscent of the flattening observed in the IMF of field stars below $0.5 M_{\odot}$ (e.g. Kroupa 2001; Chabrier 2003), also present in the mass function of L1688 pre-main sequence objects (Luhman et al. 2000; Bontemps et al. 2001). If real, the break occurs at a mass comparable to the typical Jeans mass in the dense $\left(n_{\mathrm{H} 2} \sim 10^{5} \mathrm{~cm}^{-3}\right) \mathrm{DCO}^{+}$cores of the central Ophiuchus molecular cloud (cf. Loren et al. 1990). The results of MAN98 in L1688 were essentially confirmed by independent (sub)-millimeter dust continuum surveys of the same region with SCUBA on JCMT (Johnstone et al. 2000) and SIMBA on SEST (Stanke et al. 2006).

Such a close resemblance of their mass spectrum to the IMF, in both shape and mass scale, suggests that the starless condensations identified by MAN98 are about to form stars on a one-to-one or perhaps one-to-two basis, with a high local efficiency, i.e., $M_{\star} / M_{\text {pre }} \gtrsim 50 \%$. This strongly supports scenarios according to which the bulk of the IMF is at least partly determined by pre-collapse cloud fragmentation (e.g. Larson 1985, 2005; Elmegreen 1997; Padoan \& Nordlund 2002). The problem of the origin of the IMF may thus partly reduce to a good understanding of the processes responsible for the formation and evolution of prestellar condensations. Additional processes are likely to be required, however, to account for the formation of binary/multiple systems and fully explain the lowmass $\left(M<0.3 M_{\odot}\right)$ end of the IMF. Indeed, while most young stars are observed to be in close multiple systems (e.g. Duchêne et al. 2004), the $1.2 \mathrm{~mm}$ continuum survey of MAN98 did not have enough spatial resolution to probe multiplicity within the L1688 condensations. Furthermore, multiple systems are believed to form after the prestellar stage by subsequent dynamical fragmentation during the collapse phase, close to the time of protostar formation (e.g. Goodwin et al. 2007). One would thus expect the masses of the Ophiuchus prestellar condensations to be more directly related to the masses of multiple systems than to the masses of individual stars. Surprisingly, the shape of the condensation mass spectrum agrees better with the IMF of individual field stars (solid curve in Fig. 1) than with the IMF of multiple systems (dashed curve in Fig. 1). Clearly, the link between the condensation mass spectrum and the IMF is less robust at the low-mass end than at the high-mass end.

On the theoretical side, two main scenarios have been proposed for clustered star formation in turbulent molecular clouds. In the first scenario, the distribution of stellar masses is primarily determined by gravoturbulent cloud fragmentation at the prestellar stage. Briefly, self-gravitating condensations form as turbulence-generated density fluctuations (e.g. Klessen \& Burkert 2000; Padoan \& Nordlund 2002), then decouple from their turbulent environment through the dissipation of MHD waves on small scales (e.g. Nakano 1998; Myers 1998), and eventually collapse with little interaction with their surroundings. Such protocluster condensations are local minima of turbulence (traced by narrow linewidths) and have small relative motions with respect to one another and to the surrounding gas. A given star is entirely formed from (a fraction of) the gas that was initially present in the corresponding prestellar condensation. Thus, in this scenario, the IMF derives directly from the condensation mass distribution (CMD) (Padoan \& Nordlund 2002), which provides a simple explanation for the observed similarity between the CMD and the IMF (e.g. MAN98 and Fig. 1).

By contrast, in the second scenario, the distribution of stellar masses results entirely from the dynamics of the parent protocluster (e.g. Bonnell et al. 1998, 2001b). Here, a protocluster is initially made up of gas and protostellar seeds. These protostellar seeds result from turbulence-generated cloud structure like in the first scenario, but their initial masses are unrelated to final stellar masses. The seeds travel in the gravitational potential well of the system and are characterized by a large, essentially virial velocity dispersion. They accrete mass competitively as they execute several orbits through the protocluster. The seed trajectories within the protocluster are highly stochastic in nature and feature close encounters, merging and/or dynamical ejections. In this alternative scenario, competitive accretion and dynamical interactions between individual protocluster members play a dominant role in shaping the resulting IMF at the protostellar (Class 0/Class I) stage (e.g. Bate et al. 2003). Furthermore, most of the final mass of a given star comes from gas that was initially not gravitationally bound to the corresponding protostellar seed(s) (cf. Bonnell et al. 2004).

In an effort to discriminate between these two broad pictures for the origin of the IMF and further constrain the nature of the starless condensations identified by MAN98, we carried out detailed molecular line observations of the central Ophiuchus protocluster with the IRAM $30 \mathrm{~m}$ telescope. The present paper describes the results of these line observations and discusses them in the context of the above-mentioned theoretical scenarios for clustered star formation.

The layout of the paper is as follows. Section 2 provides observational details. Section 3 presents the results of our line mapping observations. Section 4 analyzes the constraints set by these observations on the kinematics of the L1688 protocluster. We discuss the implications of our results for our understanding of cluster-forming clouds in Sect. 5. Our conclusions are summarized in Sect. 6. 
Table 1. Adopted line rest frequencies and telescope efficiencies.

\begin{tabular}{lccccccc}
\hline \hline Transition & $\begin{array}{c}\text { Frequency } \\
(\mathrm{MHz})\end{array}$ & $\begin{array}{c}\sigma_{v}^{b} \\
\left(\mathrm{~km} \mathrm{~s}^{-1}\right)\end{array}$ & $\begin{array}{c}\text { Ref. }^{c} \\
H P B W^{d} \\
\left({ }^{\prime \prime}\right)\end{array}$ & $F_{\text {eff }}^{e}$ & $\begin{array}{c}B_{\text {eff }}^{f} \\
(2000)\end{array}$ & $\begin{array}{c}B_{\text {eff }} \\
(1998)\end{array}$ \\
\hline $\mathrm{H}^{13} \mathrm{CO}^{+}(1-0)$ & $86754.294(30)$ & 0.10 & $(1)$ & 28.4 & 0.92 & 0.77 & \\
$\mathrm{~N}_{2} \mathrm{H}^{+}(101-012)$ & $93176.265(7)$ & 0.023 & $(2)$ & 26.4 & 0.92 & 0.77 & 0.73 \\
$\mathrm{C}^{34} \mathrm{~S}(2-1)$ & $96412.952(1)$ & 0.003 & $(3)$ & 25.5 & 0.92 & 0.77 & 0.73 \\
$\mathrm{CS}(2-1)$ & $97980.953(1)$ & 0.003 & $(3)$ & 25.1 & 0.92 & 0.80 & 0.73 \\
$\mathrm{H}_{2} \mathrm{CO}\left(2_{12}-1_{11}\right)$ & $140839.518(7)$ & 0.015 & $(1)$ & 17.5 & 0.90 & 0.65 & 0.54 \\
$\mathrm{DCO}^{+}(2-1)$ & $144077.319(50)$ & 0.10 & $(4)$ & 17.1 & 0.90 & 0.65 & \\
$\mathrm{CS}(3-2)$ & $146969.026(1)$ & 0.002 & $(3)$ & 16.7 & 0.90 & 0.65 & \\
$\mathrm{HCO}^{+}(3-2)$ & $267557.625(17)$ & 0.019 & $(1)$ & 9.2 & 0.85 & 0.49 & \\
\hline
\end{tabular}

${ }^{a}$ The frequency uncertainty in units of the last digit is given in parentheses.

${ }^{b}$ Frequency uncertainty converted to velocity units.

${ }^{c}$ Reference for the frequency: (1) Lovas (1992), (2) Caselli et al. (1995), (3) Gottlieb et al. (2003), (4) Pickett et al. (1998).

${ }^{d}$ Half-power beamwidth $(H P B W)$ of the IRAM $30 \mathrm{~m}$ telescope.

${ }^{e}$ The forward efficiency was 0.95 for $\mathrm{N}_{2} \mathrm{H}^{+}(1-0)$ in 2005 (OTF map in Oph B1).

${ }^{f}$ The main-beam efficiency was 0.77 for $\mathrm{N}_{2} \mathrm{H}^{+}(1-0)$ in 2005 .

\section{Observations}

We used the IRAM $30 \mathrm{~m}$ telescope at Pico Veleta, Spain, in June 1998, July 2000, and June 2005 to carry out millimeter line observations of the $\mathrm{DCO}^{+}$cores and $1.2 \mathrm{~mm}$ continuum condensations of L1688 in the following molecular transitions: $\mathrm{N}_{2} \mathrm{H}^{+}(1-0), \mathrm{H}^{13} \mathrm{CO}^{+}(1-0), \mathrm{CS}(2-1), \mathrm{C}^{34} \mathrm{~S}(2-1)$ at $3 \mathrm{~mm}$, $\mathrm{CS}(3-2), \quad \mathrm{H}_{2} \mathrm{CO}\left(2_{12}-1_{11}\right), \quad \mathrm{DCO}^{+}(2-1)$ at $2 \mathrm{~mm}$, and $\mathrm{HCO}^{+}(3-2)$ at $1.1 \mathrm{~mm}$. Our adopted set of rest line frequencies is given in Table 1. The half-power beamwidth of the telescope was $\sim 26^{\prime \prime}, \sim 17^{\prime \prime}$, and $\sim 9^{\prime \prime}$ at $3 \mathrm{~mm}, 2 \mathrm{~mm}$, and $1.1 \mathrm{~mm}$, respectively. We used four SIS heterodyne receivers simultaneously and an autocorrelation spectrometer as backend, with a spectral resolution of $20-40 \mathrm{kHz}$ at $3 \mathrm{~mm}$ and $2 \mathrm{~mm}$, and $40 \mathrm{kHz}$ at $1.1 \mathrm{~mm}$. The corresponding velocity resolution ranged from 0.04 to $0.07 \mathrm{~km} \mathrm{~s}^{-1}$ per channel, depending on the observed transition. All our observations were performed in single sideband mode, with sideband rejections of $0.01,0.1$ and 0.05 at $3 \mathrm{~mm}, 2 \mathrm{~mm}$ and $1.1 \mathrm{~mm}$, respectively. The resulting calibration uncertainty is $\sim 10 \%$. The forward and beam efficiencies of the telescope used to convert antenna temperatures $T_{\mathrm{A}}^{*}$ into main beam temperatures $T_{\mathrm{mb}}$ are listed in Table 1 . The telescope pointing was checked every $\sim 2 \mathrm{~h}$ on NRAO 530 and/or 1514-241 and found to be accurate to $\sim 4^{\prime \prime}$ (rms). The telescope focus was optimized on 3C273 and NRAO 530 every $\sim 2 \mathrm{~h}$ on average. Single-point line observations were performed in the position switching mode, while extensive mapping was performed in the "on-the-fly" (OTF) mode. All of the data were reduced with the CLASS software package".

\section{Molecular line mapping results and analysis}

\subsection{Detections of protocluster condensations in $\mathrm{N}_{2} \mathrm{H}^{+}(1-0)$ and other tracers}

The $\mathrm{N}_{2} \mathrm{H}^{+}(1-0)$ integrated intensity maps ${ }^{2}$ we took toward the six main $\mathrm{DCO}^{+}$cores of the Ophiuchus central cloud are shown in Fig. 2. The positions of the starless $1.2 \mathrm{~mm}$ continuum condensations found by MAN98 are marked by crosses. $\mathrm{N}_{2} \mathrm{H}^{+}(1-0)$ emission was found to be present toward most of these condensations. Altogether, we performed $\mathrm{N}_{2} \mathrm{H}^{+}(1-0)$ observations

\footnotetext{
1 See http: //www . iram. fr/IRAMFR/GILDAS

2 Our $\mathrm{N}_{2} \mathrm{H}^{+}(1-0)$ data cubes are available in FITS format at the CDS.
}

toward 48 of the 57 compact starless condensations ${ }^{3}$ identified by MAN98 and detected line emission for 41 of them, either through position-switch integrations $\left(\mathrm{rms} \sim 0.1 \mathrm{~K}\right.$ in $T_{\mathrm{A}}^{*}$ units) or in OTF maps ( $\mathrm{rms} \sim 0.1-0.2 \mathrm{~K}$, depending on core). However, the $\sim 26^{\prime \prime}$ angular resolution of our $\mathrm{N}_{2} \mathrm{H}^{+}$observations is not always sufficient to clearly distinguish between line emission from the condensations themselves and emission from the parent cloud $/ \mathrm{DCO}^{+}$core. In order to estimate the background $\mathrm{N}_{2} \mathrm{H}^{+}$emission from the parent core, we used our OTF maps to produce a smoothed $\mathrm{N}_{2} \mathrm{H}^{+}(1-0)$ image of the local background toward each object. In practice, we used the multiresolution wavelet analysis software of Starck et al. (1998) to decompose our OTF $\mathrm{N}_{2} \mathrm{H}^{+}(1-0)$ data cubes into two wavelet "views", corresponding to small $\left(\sim 20-40^{\prime \prime}\right)$ and large $\left(\gtrsim 40^{\prime \prime}\right)$ spatial scales, respectively. The background emission was estimated from the "large-scale" view, while an estimate of the background-subtracted emission from the condensations was provided by the "small-scale" view. Such a multi-resolution decomposition is similar to the analysis performed by MAN98 to separate compact condensations and protostellar envelopes seen on angular scales $\$ 30-60^{\prime \prime}$ from more extended $\left(\gtrsim 1^{\prime}\right)$ cloud structure in their $1.2 \mathrm{~mm}$ dust continuum image. The fact that most of the condensations detected in OTF maps remained positively detected after background subtraction demonstrates that our $\mathrm{N}_{2} \mathrm{H}^{+}$observations successfully probed the condensations themselves and not only the dense environment of the parent clump $/ \mathrm{DCO}^{+}$cores.

Additional $\mathrm{H}^{13} \mathrm{CO}^{+}(1-0)$ and $\mathrm{DCO}^{+}(2-1)$ OTF maps were obtained toward some of the cores (cf. Fig. 3), which were background-subtracted and analyzed in a similar fashion.

At the position of each target condensation/protostar, the seven hyperfine components of the $\mathrm{N}_{2} \mathrm{H}^{+}(1-0)$ multiplet were fitted simultaneously using the Gaussian HFS (HyperFine Structure) fitting routine of the CLASS software package. This routine derives the line optical depth by assuming the same excitation temperature for all hyperfine components, and therefore yields an estimate of the intrinsic linewidth (i.e., properly corrected for optical depth effects provided that the assumption is correct). The results of these HFS fits, both before and after background subtraction, are given in Table 2 for all the objects detected in $\mathrm{N}_{2} \mathrm{H}^{+}(1-0)$. (Note that, in some cases, the

\footnotetext{
3 MAN98 counted 58 compact starless condensations but one of these - E-MM3 - was subsequently shown to be an edge-on T Tauri disk as opposed to a prestellar object (e.g. Brandner et al. 2000).
} 

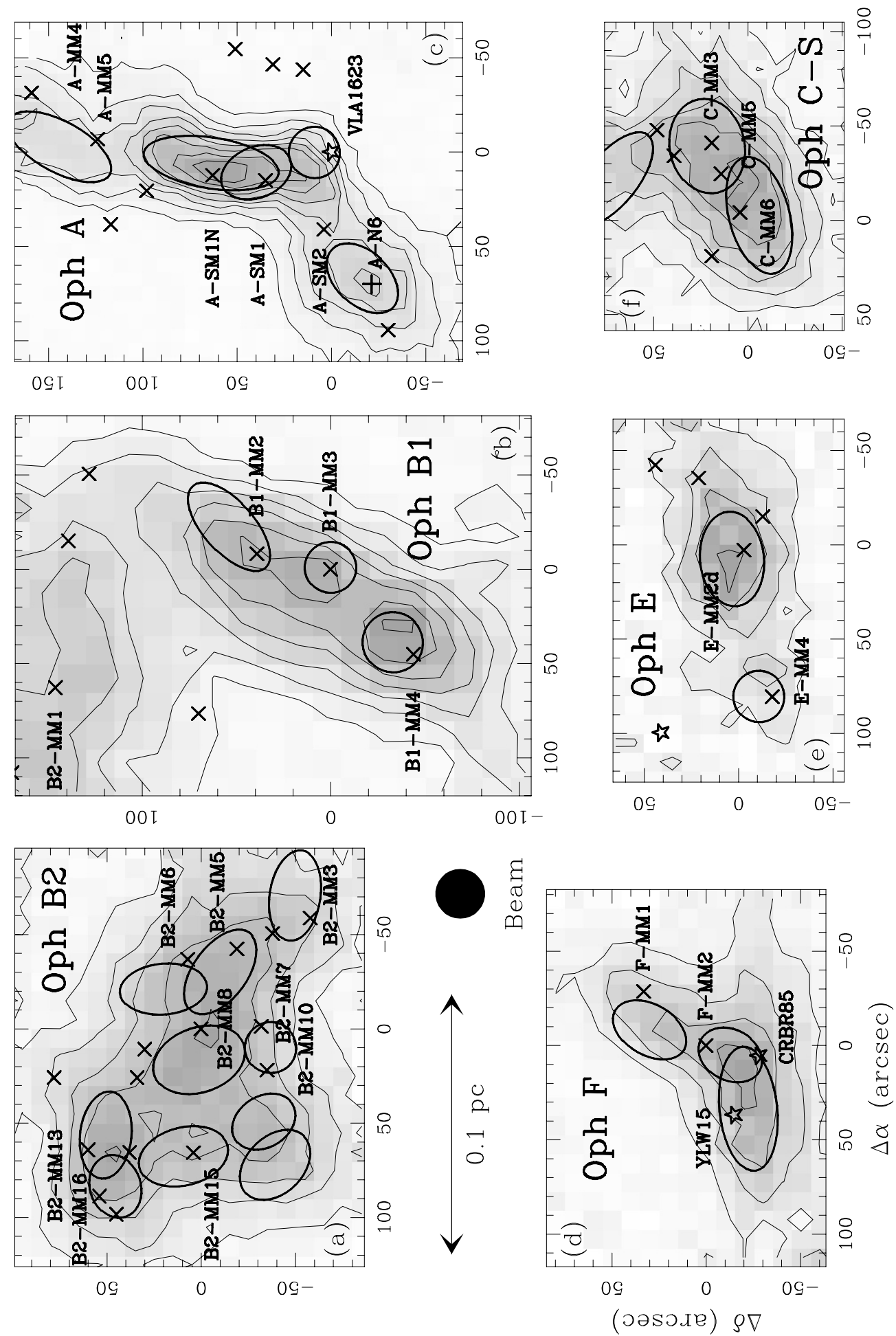

Fig. 2. Maps of the $\mathrm{N}_{2} \mathrm{H}^{+}(1-0)$ intensity, integrated over the seven components of the hyperfine multiplet, taken in the OTF mode with the IRAM 30 m telescope toward the DCO ${ }^{+}$cores a) Oph B2, b) Oph B1, c) Oph A, d) Oph F, e) Oph E, and f) Oph C-S. The $(0,0)$ offsets correspond to the J2000 equatorial positions $\left(16^{\mathrm{h}} 27^{\mathrm{m}} 27^{\mathrm{s}} .96,-24^{\circ} 27^{\prime} 06.9^{\prime \prime}\right),\left(16^{\mathrm{h}} 27^{\mathrm{m}} 12^{\mathrm{s}} .41,-24^{\circ} 29^{\prime} 58.0^{\prime \prime}\right),\left(16^{\mathrm{h}} 26^{\mathrm{m}} 26^{\mathrm{s}} 45,-24^{\circ} 24^{\prime} 30.8^{\prime \prime}\right),\left(16^{\mathrm{h}} 27^{\mathrm{m}} 24^{\mathrm{s}} .25\right.$, $\left.-24^{\circ} 40^{\prime} 35.2^{\prime \prime}\right),\left(16^{\mathrm{h}} 27^{\mathrm{m}} 04^{\mathrm{s}} .70,-24^{\circ} 39^{\prime} 12.5^{\prime \prime}\right)$, and $\left(16^{\mathrm{h}} 27^{\mathrm{m}} 01^{\mathrm{s}} .91,-24^{\circ} 34^{\prime} 40.7^{\prime \prime}\right)$, respectively. The contours go from 2 to 10,1 to 7,2 to 16 , 1.5 to $6,0.75$ to 3 , and 1 to $6 \mathrm{~K} \mathrm{~km} \mathrm{~s}^{-1}$ by steps of $2,1,2,1.5,0.75$, and $1 \mathrm{~K} \mathrm{~km} \mathrm{~s}^{-1}$, respectively (in $T_{\mathrm{a}}^{\star}$ scale). The angular resolution (HPBW) is shown as a black filled circle. The crosses mark the positions of the starless condensations identified by MAN98 in the dust continuum at $1.2 \mathrm{~mm}$, the plus symbol the position of the $\mathrm{N}_{2} \mathrm{H}^{+}$peak N6 discussed by Di Francesco et al. (2004), and the star symbols the positions of Class 0 or Class I protostars. The ellipses show the locations of the clumps identified with Gaussclumps in the corresponding $\mathrm{N}_{2} \mathrm{H}^{+}(101-012)$ background-subtracted data cubes (see Sect. 3.1 and Table 3).

signal-to-noise ratio was not good enough to perform a significant HFS fit after background subtraction.) The quoted error bars correspond to standard deviations $(1 \sigma)$ as estimated by the HFS routine of CLASS. Examples of $\mathrm{N}_{2} \mathrm{H}^{+}(1-0)$ spectra and HFS fits are shown in Fig. 4. A single-component HFS fit failed and a two-component HFS fit was required for three condensations 

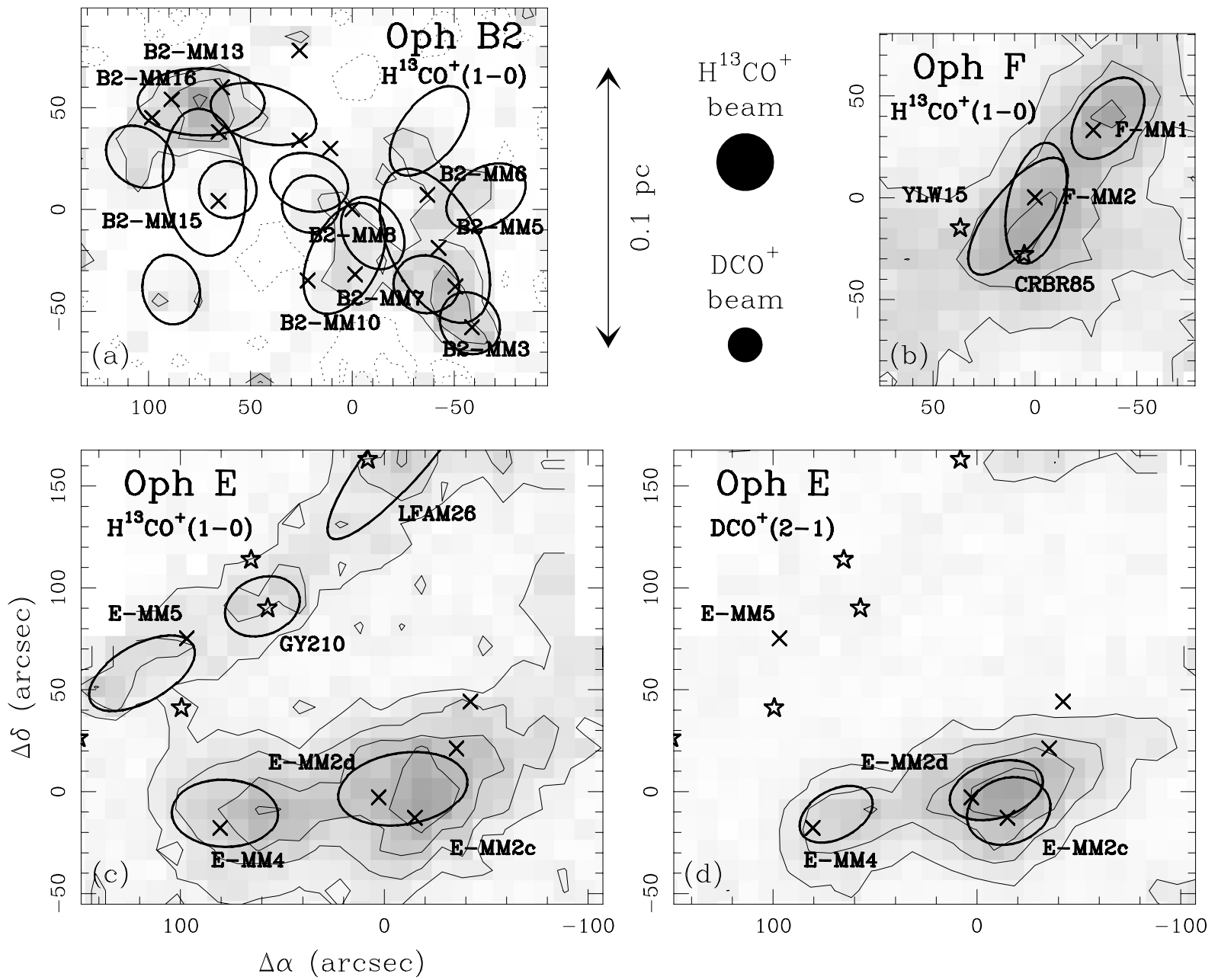

Fig. 3. $\mathrm{H}^{13} \mathrm{CO}^{+}(1-0)$ or $\mathrm{DCO}^{+}(2-1)$ integrated intensity maps taken in the OTF mode with the IRAM $30 \mathrm{~m}$ telescope toward the DCO ${ }^{+}$cores Oph B2 (a), Oph F (b), and Oph E (c, d). The contours go from 0.3 to $0.9,0.5$ to $1.5,0.25$ to 1 , and 0.6 to $2.4 \mathrm{~K} \mathrm{~km} \mathrm{~s}^{-1}$ by steps of $0.3,0.5,0.25$, $0.6 \mathrm{~K} \mathrm{~km} \mathrm{~s}^{-1}$, respectively (in $T_{\mathrm{a}}^{\star}$ scale). In a), the negative contours are -0.6 and $-0.3 \mathrm{~K} \mathrm{~km} \mathrm{~s}^{-1}$. To emphasize small-scale structure, large-scale $\left(>40^{\prime \prime}\right)$ emission was subtracted from the $\mathrm{H}^{13} \mathrm{CO}^{+}(1-0)$ map of Oph B2 shown in a) (see Sect. 3.1). The angular resolution (HPBW) is shown as a black filled circle. The crosses mark the positions of the starless condensations identified by MAN98 in the dust continuum at $1.2 \mathrm{~mm}$, and the star symbols the positions of Class 0 or Class I protostars. The ellipses show the locations of the clumps identified with Gaussclumps in the corresponding background-subtracted data cubes (see Sect. 3.1).

in Oph B1, four condensations in Oph B2, one condensation in Oph F, as well as the protostar CRBR 85.

In addition, we also used the Gaussclumps fitting procedure of Stutzki \& Güsten (1990) (see also Kramer et al. 1998) to decompose each of our background-subtracted OTF $\mathrm{N}_{2} \mathrm{H}^{+}(101-012), \mathrm{H}^{13} \mathrm{CO}^{+}(1-0)$, and $\mathrm{DCO}^{+}(2-1)$ data cubes into a series of Gaussian-shaped "clumps". The significant $(>5 \sigma)$ clumps identified with Gaussclumps in the various $\mathrm{DCO}^{+}$cores of L1688 are shown as ellipses in Figs. 2 and 3. Their main characteristics are given in Table 3. In this way, a total of 17 starless $1.2 \mathrm{~mm}$ condensations and 3 Class 0/Class I protostars (VLA 1623, LFAM 26, GY 210) were found to have well-defined $\mathrm{N}_{2} \mathrm{H}^{+}, \mathrm{H}^{13} \mathrm{CO}^{+}$, or $\mathrm{DCO}^{+}$counterparts in positionvelocity $(l-v)$ space. The relevance of such a Gaussclumps decomposition is that it further helped us discriminate between the line emission arising from the compact condensations themselves (with well-defined "positions" in $l-v$ space) and the line emission from the surrounding, more extended dense gas (with less-well-defined "positions" in $l-v$ space). In particular, for six of the above-mentioned eight condensations with double $\mathrm{N}_{2} \mathrm{H}^{+}$HFS components, the Gaussclumps decomposition allowed us to reject one of the two components and to identify the component most likely associated with the compact $1.2 \mathrm{~mm}$ continuum object. In Oph B1, for instance, two HFS components were detected toward B1-MM2, B1-MM3, and B1-MM4 prior to background subtraction (cf. Table 2), but only one of these two components was found to be associated with each condensation after running Gaussclumps on the backgroundsubtracted $\mathrm{N}_{2} \mathrm{H}^{+}(101-012)$ data cubes (cf. Table 3). In the following, we will consider the multiple components detected toward the remaining two condensations with double HFS components (B2-MM2 and B2-MM4) as representative of independent objects when we discuss the statistics of linewidths (Sect. 3.2) and relative motions (Sect. 4.3) between condensations.

\subsection{Linewidths and virial masses of the protocluster condensations}

Based on the measured $\mathrm{N}_{2} \mathrm{H}^{+}(1-0)$ linewidths (Table 2), we can calculate the nonthermal component of the line-of-sight velocity 
Table 2. Results of Gaussian hyperfine fits to the $\mathrm{N}_{2} \mathrm{H}^{+}(1-0)$ multiplet.

\begin{tabular}{|c|c|c|c|c|c|c|c|c|c|c|c|c|c|c|}
\hline \multirow{3}{*}{ Source } & \multirow{2}{*}{\multicolumn{2}{|c|}{ Coordinates }} & \multicolumn{6}{|c|}{ Prior to background subtraction } & \multicolumn{6}{|c|}{ After background subtraction } \\
\hline & & & \multirow{2}{*}{\multicolumn{2}{|c|}{$\begin{array}{l}\sigma^{a} S / N^{b} \\
(\mathrm{mK})\end{array}$}} & \multirow{2}{*}{$\begin{array}{l}P_{1}^{c} \\
(\mathrm{~K})\end{array}$} & \multirow{2}{*}{$\begin{array}{c}V_{\mathrm{lsr}} \\
\left(\mathrm{km} \mathrm{s}^{-1}\right)\end{array}$} & \multirow{2}{*}{$\begin{array}{l}F W H M \\
\left(\mathrm{~km} \mathrm{~s}^{-1}\right)\end{array}$} & \multirow[t]{2}{*}{$\tau_{\text {tot }}^{d}$} & \multirow{2}{*}{\multicolumn{2}{|c|}{$\begin{array}{l}\sigma^{a} S / N^{b} \\
(\mathrm{mK})\end{array}$}} & \multirow{2}{*}{$\begin{array}{l}P_{1}^{c} \\
(\mathrm{~K})\end{array}$} & $V_{\mathrm{lsr}}$ & $F W H M$ & $\tau_{\text {tot }}^{d}$ \\
\hline & $\alpha_{2000}$ & $\delta_{2000}$ & & & & & & & & & & $\left(\mathrm{~km} \mathrm{~s}^{-1}\right)$ & $\left(\mathrm{km} \mathrm{s}^{-1}\right)$ & \\
\hline (1) & $\begin{array}{c}16^{\mathrm{h}} \\
(2)\end{array}$ & $\begin{array}{r}-24^{\circ} \\
(3)\end{array}$ & (4) & (5) & (6) & (7) & (8) & (9) & (10) & (11) & (12) & (13) & (14) & (15) \\
\hline A3-MM1 & $26: 09.7$ & $23: 06$ & 79 & 7 & $1.6(2)$ & $3.243(26)$ & $0.877(59)$ & $0.2(24)$ & & & & & & \\
\hline A-MM4 & $26: 24.1$ & $21: 52$ & 65 & 45 & $15.8(1)$ & $3.243(2)$ & $0.445(2)$ & $2.6(1)$ & 67 & 17 & $4.5(5)$ & $3.324(8)$ & $0.378(18)$ & $1.5(13)$ \\
\hline A-MM5 & $26: 25.9$ & $22: 27$ & 62 & 53 & $15.3(5)$ & $3.171(2)$ & $0.381(6)$ & $1.9(3)$ & 64 & 15 & $9.8(16)$ & $3.146(5)$ & $0.183(10)$ & $10.6(29)$ \\
\hline SM1N & $26: 27.3$ & $23: 28$ & 60 & 98 & $52.2(8)$ & $3.573(1)$ & $0.487(3)$ & $8.6(2)$ & 57 & 47 & $29.0(9)$ & $3.587(3)$ & $0.436(6)$ & $11.4(5)$ \\
\hline SM1 & $26: 27.5$ & 23:56 & 43 & 125 & $35.9(0)$ & $3.645(1)$ & $0.597(1)$ & $5.7(0)$ & 43 & 56 & $15.2(4)$ & $3.682(3)$ & $0.640(7)$ & $6.1(3)$ \\
\hline A-MM6 & $26: 27.9$ & $22: 53$ & 64 & 20 & $5.0(3)$ & $3.353(6)$ & $0.694(16)$ & $1.4(5)$ & & & & & & \\
\hline SM2 & $26: 29.5$ & $24: 27$ & 52 & 68 & $19.8(3)$ & $3.509(1)$ & $0.485(3)$ & $3.6(2)$ & 51 & 19 & $5.8(5)$ & $3.519(6)$ & $0.386(13)$ & $4.9(12)$ \\
\hline A-MM8 & $26: 33.4$ & 25:01 & 65 & 51 & $17.3(4)$ & $3.505(1)$ & $0.384(4)$ & $2.7(3)$ & 64 & 16 & $3.9(1)$ & $3.571(6)$ & $0.332(12)$ & $0.1(6)$ \\
\hline A-S & $26: 43.1$ & $25: 42$ & 81 & 7 & $2.9(6)$ & $3.710(7)$ & $0.236(20)$ & $2.2(22)$ & & & & & & \\
\hline VLA1623 & $26: 26.5$ & $24: 31$ & 37 & 75 & 17.1(2) & $3.685(1)$ & $0.561(3)$ & $4.4(1)$ & 37 & 30 & $6.9(3)$ & $3.749(4)$ & $0.532(8)$ & $4.2(5)$ \\
\hline B1-MM1 & $27: 08.7$ & $27: 50$ & 91 & 10 & $3.1(1)$ & $4.069(8)$ & $0.383(21)$ & $0.1(2)$ & & & & & & \\
\hline B1-MM2 & $27: 11.8$ & $29: 19$ & 101 & 26 & $5.4(5)$ & $3.387(19)$ & $0.826(39)$ & $2.2(7)$ & 100 & 11 & $1.9(2)$ & $3.430(42)$ & 0.981(88) & $0.1(203)$ \\
\hline & & & & & $10.2(8)$ & $4.050(4)$ & $0.287(11)$ & $3.2(9)$ & & & $4.8(10)$ & $4.060(8)$ & $0.217(26)$ & $5.1(28)$ \\
\hline B1-MM3 & $27: 12.4$ & $29: 58$ & 67 & 38 & $9.7(8)$ & $3.287(4)$ & $0.280(11)$ & $11.1(15)$ & & & & & & \\
\hline & & & & & $14.6(4)$ & $3.821(2)$ & $0.340(5)$ & $3.3(4)$ & 70 & 19 & $16.9(22)$ & $3.780(3)$ & $0.185(7)$ & $13.7(27)$ \\
\hline B1-MM4 & $27: 15.7$ & $30: 42$ & 216 & 15 & $34.8(68)$ & $3.551(5)$ & $0.158(11)$ & $23.5(54)$ & 221 & 8 & $14.8(14)$ & $3.762(27)$ & $0.451(37)$ & $29.8(37)$ \\
\hline & & & & & $14.6(12)$ & $3.956(5)$ & $0.341(14)$ & $2.2(10)$ & & & $4.3(7)$ & $3.967(11)$ & $0.205(22)$ & $0.3(13)$ \\
\hline B1B2-MM1 & $27: 11.3$ & $27: 39$ & 130 & 16 & $7.6(7)$ & $4.070(6)$ & $0.384(16)$ & $0.2(12)$ & 128 & 5 & $3.0(11)$ & $4.065(12)$ & $0.217(39)$ & $1.5(37)$ \\
\hline B1B2-MM2 ${ }^{e}$ & $27: 18.0$ & $28: 48$ & 126 & 6 & $2.7(6)$ & $3.957(25)$ & $0.556(63)$ & $2.4(25)$ & & & & & & \\
\hline B2-MM1 & $27: 17.0$ & $27: 32$ & 147 & 16 & $12.6(11)$ & $4.049(6)$ & $0.380(14)$ & $4.5(11)$ & 144 & 4 & $6.2(37)$ & $3.984(15)$ & $0.184(42)$ & 15.1(114) \\
\hline B2-MM2 & $27: 20.3$ & 27:08 & 99 & 18 & $7.5(2)$ & $3.947(19)$ & $0.757(34)$ & $3.7(4)$ & & & & & & \\
\hline & & & & & $6.7(7)$ & $4.344(7)$ & $0.238(18)$ & $10.3(0)$ & & & & & & \\
\hline B2-MM3 & $27: 23.7$ & 28:05 & 130 & 14 & $8.4(2)$ & $3.785(9)$ & $0.531(11)$ & $3.0(1)$ & & & & & & \\
\hline & & & & & $4.2(9)$ & $4.376(4)$ & $0.305(30)$ & $2.1(23)$ & & & & & & \\
\hline B2-MM4 & $27: 24.3$ & $27: 45$ & 262 & 14 & 21.7(19) & $3.718(7)$ & $0.393(17)$ & $6.4(13)$ & 259 & 5 & $14.9(46)$ & $3.678(12)$ & $0.262(25)$ & $16.3(66)$ \\
\hline & & & & & $4.6(7)$ & $4.358(15)$ & $0.345(38)$ & $0.1(22)$ & & & & & & \\
\hline B2-MM5 & $27: 24.9$ & $27: 26$ & 246 & 13 & $15.8(4)$ & $3.693(1)$ & $0.497(19)$ & $2.9(0)$ & 238 & 5 & $7.9(21)$ & $3.644(15)$ & $0.305(28)$ & $7.7(42)$ \\
\hline & & & & & $4.7(15)$ & $4.388(62)$ & $0.597(97)$ & $8.0(45)$ & & & & & & \\
\hline B2-MM6 & $27: 25.3$ & 27:00 & 240 & 12 & $15.6(13)$ & $3.763(10)$ & $0.699(26)$ & $4.0(8)$ & 248 & 4 & $10.1(28)$ & $3.680(22)$ & $0.424(39)$ & $14.3(56)$ \\
\hline B2-MM7 & $27: 27.9$ & 27:39 & 261 & 10 & $9.2(13)$ & $4.214(21)$ & $0.789(60)$ & $2.0(11)$ & & & & & & \\
\hline B2-MM8 & $27: 28.0$ & 27:07 & 206 & 19 & $19.2(11)$ & $4.144(6)$ & $0.584(14)$ & $3.0(6)$ & 208 & 8 & $6.3(3)$ & $4.186(10)$ & $0.419(17)$ & $0.1(5)$ \\
\hline B2-MM9 & $27: 28.8$ & $26: 37$ & 240 & 13 & $11.3(12)$ & 4.093(12) & $0.650(33)$ & $1.9(10)$ & & & & & & \\
\hline B2-MM10 & $27: 29.6$ & $27: 42$ & 162 & 15 & $8.9(8)$ & $4.334(9)$ & $0.569(22)$ & $0.5(8)$ & & & & & & \\
\hline B2-MM11 & $27: 29.8$ & $25: 49$ & 145 & 12 & $4.2(2)$ & $4.040(16)$ & $1.018(51)$ & $0.1(4)$ & 146 & 4 & $4.4(26)$ & $4.070(54)$ & $0.633(134)$ & $15.4(104)$ \\
\hline B2-MM12 & $27: 29.9$ & $26: 33$ & 244 & 14 & 16.4(12) & 4.093(8) & $0.549(20)$ & $3.5(9)$ & 255 & 4 & $8.5(24)$ & $4.097(12)$ & $0.238(26)$ & 7.7(44) \\
\hline B2-MM13 & $27: 32.7$ & $26: 07$ & 263 & 12 & 14.1(14) & $3.967(12)$ & $0.637(29)$ & $4.1(11)$ & & & & & & \\
\hline B2-MM14 & $27: 32.8$ & $26: 29$ & 258 & 11 & $14.9(14)$ & $4.162(12)$ & $0.771(32)$ & $3.8(9)$ & & & & & & \\
\hline B2-MM15 & $27: 32.8$ & 27:03 & 267 & 15 & $31.6(24)$ & $4.413(5)$ & $0.356(11)$ & 7.1(11) & 272 & 6 & $14.9(36)$ & $4.445(8)$ & $0.221(17)$ & $9.3(41)$ \\
\hline B2-MM16 & $27: 34.5$ & $26: 12$ & 55 & 54 & $15.7(2)$ & $4.041(2)$ & $0.626(4)$ & $3.5(2)$ & 54 & 23 & $9.2(7)$ & $4.074(7)$ & $0.426(15)$ & $6.8(10)$ \\
\hline B2-MM17 & $27: 35.2$ & $26: 21$ & 250 & 12 & 13.1(14) & $4.079(12)$ & $0.622(28)$ & $3.4(11)$ & 257 & 5 & $4.2(14)$ & $4.110(19)$ & $0.320(45)$ & $1.4(33)$ \\
\hline $\mathrm{C}-\mathrm{W}^{e}$ & $26: 50.0$ & $32: 49$ & 154 & 12 & $14.6(17)$ & $3.578(4)$ & $0.192(9)$ & $7.2(18)$ & & & & & & \\
\hline $\mathrm{C}-\mathrm{N}^{e}$ & $26: 57.2$ & $31: 39$ & 93 & 33 & $36.3(16)$ & 3.811(1) & $0.211(3)$ & $12.5(8)$ & & & & & & \\
\hline C-MM2 & $26: 58.4$ & $33: 53$ & 263 & 8 & $18.2(27)$ & $3.879(12)$ & $0.429(24)$ & $11.5(26)$ & & & & & & \\
\hline C-MM3 & $26: 58.9$ & $34: 22$ & 281 & 9 & $35.2(46)$ & $3.910(7)$ & $0.307(15)$ & $17.4(30)$ & & & & & & \\
\hline C-MM4 & $26: 59.4$ & 34:02 & 287 & 9 & $28.6(49)$ & $3.852(9)$ & $0.334(18)$ & $15.7(38)$ & & & & & & \\
\hline C-MM5 & 27:00.1 & $34: 27$ & 62 & 38 & $38.5(12)$ & $3.809(1)$ & $0.314(3)$ & $17.6(7)$ & 62 & 16 & $22.1(7)$ & $3.803(5)$ & $0.238(6)$ & $30.0(25)$ \\
\hline C-MM6 & 27:01.6 & $34: 37$ & 61 & 38 & $37.1(22)$ & $3.710(1)$ & $0.327(6)$ & $17.4(13)$ & 61 & 18 & $24.8(26)$ & $3.667(4)$ & $0.249(7)$ & $26.3(33)$ \\
\hline C-MM7 & 27:03.3 & $34: 22$ & 294 & 6 & $34.1(15)$ & $3.691(10)$ & $0.294(11)$ & $30.0(44)$ & & & & & & \\
\hline E-MM1 ${ }^{e}$ & $26: 57.7$ & $36: 56$ & 153 & 5 & $2.1(2)$ & $4.434(21)$ & $0.360(39)$ & $0.1(195)$ & & & & & & \\
\hline E-MM2d & 27:04.9 & $39: 15$ & 61 & 39 & $13.5(4)$ & $4.496(1)$ & $0.287(4)$ & $3.6(4)$ & 62 & 21 & $9.0(7)$ & & & $5.5(10)$ \\
\hline E-MM4 & $27: 10.6$ & $39: 30$ & 41 & 26 & $5.1(3)$ & $4.232(3)$ & $0.331(7)$ & $1.7(6)$ & 41 & 16 & $4.8(6)$ & $4.219(6)$ & $0.254(13)$ & $6.7(19)$ \\
\hline F-MM1 & $27: 22.1$ & 40:02 & 59 & 26 & $8.9(5)$ & $4.726(5)$ & $0.476(11)$ & $4.6(7)$ & 62 & 15 & $3.4(1)$ & $4.789(6)$ & $0.329(13)$ & $0.1(9)$ \\
\hline F-MM2 & $27: 24.3$ & $40: 35$ & 43 & 53 & $18.2(2)$ & $4.133(0)$ & $0.229(4)$ & $10.8(0)$ & 44 & 22 & $6.4(8)$ & $4.148(4)$ & $0.188(11)$ & $9.6(23)$ \\
\hline & & & & & $7.7(2)$ & $4.592(2)$ & $0.354(6)$ & $0.6(3)$ & & & $4.6(4)$ & $4.584(1)$ & $0.304(11)$ & $2.0(10)$ \\
\hline CRBR85 & $27: 24.7$ & 41:03 & 215 & 13 & $25.4(26)$ & $4.067(4)$ & $0.230(10)$ & $10.2(17)$ & 213 & 6 & $12.3(28)$ & $4.032(8)$ & $0.200(19)$ & $11.1(41)$ \\
\hline & & & & & $3.3(3)$ & $4.649(20)$ & $0.402(44)$ & $0.1(4)$ & & & & & & \\
\hline YLW15 & $27: 27.0$ & 40:50 & 31 & 94 & $29.5(4)$ & $4.162(1)$ & $0.258(2)$ & $8.1(2)$ & 36 & 54 & $19.0(10)$ & $4.172(2)$ & $0.212(4)$ & $10.1(9)$ \\
\hline
\end{tabular}

Notes: the numbers in parentheses indicate the uncertainty in units of the last digit.

${ }^{a} \sigma$ is the rms noise in $T_{a}^{\star}$ scale.

${ }^{b} \mathrm{~S} / \mathrm{N}$ is the signal-to-noise ratio

${ }^{c}$ The fitting function is $T_{\mathrm{a}}^{\star}(v)=\frac{P_{1}}{\tau_{\text {tot }}}\left(1-\mathrm{e}^{-\tau(v)}\right)$. In the optically thick case, $P_{1}=T_{\mathrm{a}}^{\star \text {, peak }} \times \tau_{\text {tot }}$.

${ }^{d} \tau_{\text {tot }}$ is the total optical depth of the $\mathrm{N}_{2} \mathrm{H}^{+}(1-0)$ multiplet. The optical depth of the isolated component $101-012$ is $\frac{\tau_{\text {tot }}}{9}$.

${ }^{e}$ Composite starless clump (cf. MAN98) not included in the condensation mass distributions shown in Figs. 1 and 8. 
Table 3. Compact objects identified with Gaussclumps in the background-subtracted $\mathrm{N}_{2} \mathrm{H}^{+}(101-012)$ data cubes.

\begin{tabular}{|c|c|c|c|c|c|c|c|c|c|c|}
\hline $\begin{array}{l}\text { Object } \\
\text { name } \\
\text { (1) }\end{array}$ & $\begin{array}{l}\Delta \alpha \\
\left({ }^{\prime \prime}\right) \\
(2)\end{array}$ & $\begin{array}{l}\Delta \delta \\
\left({ }^{\prime \prime}\right) \\
(3)\end{array}$ & $\begin{array}{c}v_{0} \\
\left(\mathrm{~km} \mathrm{~s}^{-1}\right) \\
(4)\end{array}$ & $\begin{array}{l}a_{0} \\
(\mathrm{~K}) \\
(5)\end{array}$ & $\begin{array}{l}\Delta x \\
\left({ }^{\prime \prime}\right) \\
(6)\end{array}$ & $\begin{array}{l}\Delta y \\
\left({ }^{\prime \prime}\right) \\
(7)\end{array}$ & $\begin{array}{l}\phi \\
\left({ }^{\circ}\right) \\
(8)\end{array}$ & $\begin{array}{c}F W H M \\
\left({ }^{\prime \prime}\right) \\
(9)\end{array}$ & $\begin{array}{c}\Delta v \\
\left(\mathrm{~km} \mathrm{~s}^{-1}\right) \\
(10)\end{array}$ & Identification \\
\hline $\mathrm{A}-1$ & 6.0 & 63.6 & 3.52 & 2.16 & 27.00 & 72.50 & 81.2 & 35.8 & 0.49 & A-SM1N \\
\hline A-2 & 67.1 & -16.6 & 3.42 & 1.85 & 28.40 & 44.70 & 46.6 & 33.9 & 0.37 & $\mathrm{~A}-\mathrm{N} 6^{a}$ \\
\hline A-3 & -2.6 & 144.1 & 3.14 & 1.01 & 27.00 & 60.90 & 61.5 & 34.9 & 0.34 & A-MM5 \\
\hline A-6 & 10.9 & 39.7 & 3.90 & 0.65 & 27.00 & 38.70 & 112.1 & 31.3 & 0.22 & A-SM1 \\
\hline A-15 & 0.4 & 8.9 & 3.89 & 0.35 & 27.00 & 27.30 & 25.1 & 27.1 & 0.13 & VLA 1623 \\
\hline B1-1 & 38.9 & -33.0 & 4.00 & 0.73 & 31.80 & 32.20 & 50.6 & 32.0 & 0.37 & B1-MM4 \\
\hline B1-4 & -0.8 & 0.0 & 3.75 & 0.60 & 27.00 & 27.40 & 86.3 & 27.2 & 0.25 & B1-MM3 \\
\hline B1-20 & -22.3 & 53.8 & 4.24 & 0.45 & 27.00 & 58.10 & 41.8 & 34.6 & 0.15 & B1-MM2 \\
\hline $\bar{B} 2-1$ & 67.8 & 9.3 & 4.43 & 1.09 & 30.80 & 48.20 & $\overline{104.2}$ & 36.7 & 0.29 & B2-MM15 \\
\hline B2-2 & 56.5 & 50.5 & 3.91 & 0.92 & 46.40 & 27.30 & -6.3 & 33.2 & 0.35 & B2-MM13 \\
\hline B2-3 & 16.6 & 1.0 & 4.17 & 0.78 & 34.40 & 50.20 & 109.2 & 40.1 & 0.31 & B2-MM8 \\
\hline B2-4 & 72.3 & -39.3 & 4.02 & 0.77 & 30.20 & 43.20 & 134.7 & 35.0 & 0.36 & \\
\hline B2-5 & -30.0 & -10.2 & 3.76 & 0.66 & 51.70 & 28.60 & -36.5 & 35.4 & 0.37 & B2-MM5 \\
\hline B2-6 & 49.4 & -33.3 & 3.53 & 0.54 & 27.10 & 35.80 & 121.9 & 30.6 & 0.46 & \\
\hline B2-7 & -20.9 & 19.9 & 3.41 & 0.49 & 27.00 & 46.40 & 94.9 & 33.0 & 0.24 & \\
\hline B2-8 & 83.7 & 45.4 & 4.16 & 0.51 & 27.00 & 33.90 & 17.7 & 29.9 & 0.28 & B2-MM16,17 \\
\hline B2-10 & -70.5 & -49.9 & 3.79 & 0.45 & 27.00 & 48.40 & 171.4 & 33.4 & 0.22 & B2-MM3 \\
\hline B2-11 & 10.1 & -36.9 & 4.41 & 0.45 & 27.00 & 27.30 & 78.7 & 27.1 & 0.23 & B2-MM7,10 \\
\hline $\mathrm{C}-1$ & -2.4 & -6.2 & 3.67 & 0.93 & 63.70 & 32.10 & 15.1 & 40.5 & 0.32 & C-MM6 \\
\hline $\mathrm{C}-2$ & -39.1 & 21.4 & 3.92 & 0.76 & 50.50 & 39.80 & 11.7 & 44.2 & 0.31 & C-MM3, C-MM5 \\
\hline $\mathrm{C}-3$ & -21.5 & 72.0 & 3.78 & 0.42 & 27.00 & 60.70 & 140.6 & 34.9 & 0.19 & \\
\hline E-1 & 7.7 & 3.3 & 4.52 & 0.61 & 50.30 & 34.00 & 5.5 & 39.8 & 0.25 & E-MM2d \\
\hline E-4 & 80.6 & -10.9 & 4.28 & 0.37 & 27.00 & 27.30 & 24.7 & 27.2 & 0.28 & E-MM4 \\
\hline F-1 & 33.7 & -22.5 & 4.15 & 0.82 & 66.10 & 30.60 & 6.0 & 39.3 & 0.24 & حYLW 15 \\
\hline F-2 & -8.1 & 29.9 & 4.54 & 0.51 & 27.00 & 42.10 & 60.3 & 32.1 & 0.26 & F-MM1 \\
\hline F-4 & 5.2 & -12.9 & 4.66 & 0.41 & 27.00 & 35.30 & 62.0 & 30.3 & 0.31 & $\sim \mathrm{F}-\mathrm{MM} 2$ \\
\hline
\end{tabular}

Notes: in $\mathrm{Col} 1$, the first part of the object name indicates the $\mathrm{DCO}^{+}$core in which the object is embedded. Columns 2 and 3 are the offsets of the object with respect to the core position given in the caption of Fig. 2. Columns 4 and 5 are the center velocity and peak intensity, respectively. Columns 6 and 7 give the major and minor angular diameters of the two-dimensional Gaussian fitted to the object, and Col. 8 is the position angle of the minor axis. Columns 9 and 10 are the typical FWHM size and FWHM line width, respectively.

${ }^{a} \mathrm{~N}_{2} \mathrm{H}^{+}$peak N6 discussed by Di Francesco et al. (2004).
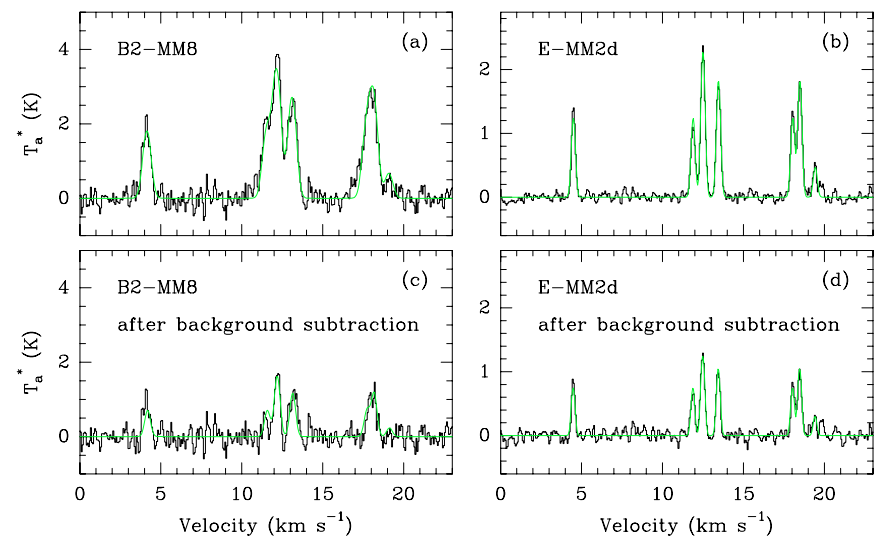

Fig. 4. Examples of $\mathrm{N}_{2} \mathrm{H}^{+}(1-0)$ spectra and Gaussian HFS fits obtained toward the condensations B2-MM8 (left) and E-MM2d (right). The spectra shown in the top row are those observed toward a) B2-MM8 and b) E-MM2d prior to background subtraction, while the spectra shown in the bottom row are those obtained toward the same sources $a f$ ter subtracting the local background emission estimated using a multiresolution wavelet decomposition (see text).

dispersion (cf. Myers 1999) and estimate a virial mass for each condensation. Table 4 gives the deconvolved (FWHM) diameter (Col. 2, MAN98) and gas + dust mass derived from the $1.2 \mathrm{~mm}$ continuum (Col. 4, MAN98), the assumed gas/dust temperature (Col. 3, cf. MAN98), the thermal velocity dispersion
$\sigma_{\mathrm{T}}(\mu)$ for a particle of mean molecular weight $\mu=2.33$ (Col. 5), the one-dimensional nonthermal velocity dispersion $\sigma_{\mathrm{NT}}$ obtained from the $\mathrm{N}_{2} \mathrm{H}^{+}(1-0)$ linewidth (Col. 6), the nonthermal to thermal velocity dispersion ratio $\sigma_{\mathrm{NT}} / \sigma_{\mathrm{T}}(\mathrm{Col} .7)$, the estimated virial mass $M_{\mathrm{vir}}$ (Col. 8), and the virial mass ratio $\alpha_{\text {vir }} \equiv M_{\text {vir }} / M_{1.2 \mathrm{~mm}}$ (Col. 9) for each observed object. Columns 10 to 13 list the values of the same parameters when estimated from the background-subtracted spectra. For some condensations, the dust temperatures (Col. 3) adopted to estimate $M_{1.2 \mathrm{~mm}}$ (Col. 4) from the measured $1.2 \mathrm{~mm}$ flux differ slightly from those assumed by MAN98. Here, we used $T_{\mathrm{d}}=12 \mathrm{~K}$ for all condensations except SM1N, SM1, A-MM6, SM2, AMM8 in Oph A, which are likely warmer $\left(T_{\mathrm{d}}=20 \mathrm{~K}\right)$ due to their proximity to the B3 star S1 (cf. André et al. 1993). Our adopted default $T_{\mathrm{d}}=12 \mathrm{~K}$ is consistent with the typical mass-averaged temperature found by dust radiative transfer models of starless cores (cf. André et al. 2003; Stamatellos \& Whitworth 2003). In agreement with the radial profiles measured by MAN98, the values quoted for $M_{\text {vir }}$ and $\alpha_{\text {vir }}$ assume that the density structure of the sources approaches that of centrallycondensed spheres with outer profiles such as $\rho \propto r^{-2}$, for which $M_{\mathrm{vir}} \approx 3 r_{\text {cond }} \frac{\sigma_{\mathrm{tot}}^{2}}{G}$. Here, $\sigma_{\mathrm{tot}}=\sqrt{\sigma_{\mathrm{T}}(\mu)^{2}+\sigma_{\mathrm{NT}}^{2}}$ is the total (thermal + nonthermal) line-of-sight velocity dispersion and $r_{\text {cond }}$ is the condensation outer radius, which we take to be twice the geometrical mean of the deconvolved major and minor $H W H M$ radii measured in the $1.2 \mathrm{~mm}$ dust continuum (i.e., $\sqrt{F W H M_{\mathrm{a}} \times F W H M_{\mathrm{b}}}-$ cf. Col. 2 of Table 4 and Table 2 of 
Table 4. Line-of-sight velocity dispersions and virial mass estimates for the 41 starless $1.2 \mathrm{~mm}$ condensations positively detected in $\mathrm{N}_{2} \mathrm{H}^{+}$.

\begin{tabular}{|c|c|c|c|c|c|c|c|c|c|c|c|c|}
\hline \multirow[b]{2}{*}{ Source } & \multirow[b]{2}{*}{$\begin{array}{c}F W H M \\
(\mathrm{AU} \times \mathrm{AU})\end{array}$} & \multirow[b]{2}{*}{$\begin{array}{l}T_{\mathrm{d}}^{b} \\
(\mathrm{~K})\end{array}$} & \multirow[b]{2}{*}{$\begin{array}{c}M_{1.2 \mathrm{~mm}^{b}} \\
\left(M_{\odot}\right)\end{array}$} & \multirow[b]{2}{*}{$\begin{array}{c}\sigma_{\mathrm{T}}(\mu) \\
\left(\mathrm{km} \mathrm{s}^{-1}\right) \\
(5)\end{array}$} & \multicolumn{4}{|c|}{ Prior to background subtraction } & \multicolumn{4}{|c|}{ After background subtraction } \\
\hline & & & & & $\begin{array}{c}\sigma_{\mathrm{NT}} \\
\left(\mathrm{km} \mathrm{s}^{-1}\right) \\
(6)\end{array}$ & $\begin{array}{l}\frac{\sigma_{\mathrm{NT}}}{\sigma_{\mathrm{T}}} \\
(7)\end{array}$ & $\begin{array}{c}M_{\mathrm{vir}} \\
\left(M_{\odot}\right) \\
(8)\end{array}$ & $\begin{array}{c}\frac{M_{\mathrm{vir}}}{M_{1.2 \mathrm{~mm}}} \\
(9)\end{array}$ & $\begin{array}{c}\sigma_{\mathrm{NT}} \\
\left(\mathrm{km} \mathrm{s}^{-1}\right) \\
(10)\end{array}$ & $\begin{array}{l}\frac{\sigma_{\mathrm{NT}}}{\sigma_{\mathrm{T}}} \\
(11)\end{array}$ & $\begin{array}{c}M_{\mathrm{vir}} \\
\left(M_{\odot}\right) \\
(12)\end{array}$ & $\begin{array}{c}\frac{M_{\mathrm{vir}}}{M_{1.2 \mathrm{~mm}}} \\
(13)\end{array}$ \\
\hline A3-MM1 & $<400$ & 12 & 0.21 & 0.21 & 0.37 & 1.8 & $<0.24$ & $<1.2$ & & & & \\
\hline A-MM4 & $4000 \times 1400$ & 12 & 0.29 & 0.21 & 0.18 & 0.9 & 0.60 & 2.1 & 0.15 & 0.7 & 0.52 & 1.8 \\
\hline A-MM5 & $3700 \times 2900$ & 12 & 0.48 & 0.21 & 0.15 & 0.7 & 0.72 & 1.5 & 0.05 & 0.2 & 0.50 & 1.1 \\
\hline SM1N & $3000 \times 1800$ & 20 & 1.30 & 0.27 & 0.19 & 0.7 & 0.85 & 0.7 & 0.17 & 0.6 & 0.78 & 0.6 \\
\hline SM1 & $6100 \times 2100$ & 20 & 3.20 & 0.27 & 0.24 & 0.9 & 1.57 & 0.5 & 0.26 & 1.0 & 1.68 & 0.5 \\
\hline A-MM6 & $3200 \times 2700$ & 20 & 0.40 & 0.27 & 0.28 & 1.1 & 1.51 & 3.8 & & & & \\
\hline SM2 & $6200 \times 3400$ & 20 & 1.30 & 0.27 & 0.19 & 0.7 & 1.67 & 1.3 & 0.15 & 0.5 & 1.43 & 1.1 \\
\hline A-MM8 & $2900 \times 2100$ & 20 & 0.13 & 0.27 & 0.14 & 0.5 & 0.77 & 5.9 & 0.12 & 0.4 & 0.71 & 5.5 \\
\hline A-S & $<400$ & 12 & 0.17 & 0.21 & 0.08 & 0.4 & $<0.07$ & $<0.4$ & & & & \\
\hline B1-MM1 & $<400$ & 12 & 0.10 & 0.21 & 0.15 & 0.7 & $<0.09$ & $<0.9$ & & & & \\
\hline $\mathrm{B} 1-\mathrm{MM} 2^{a}$ & $3000 \times 2100$ & 12 & 0.17 & 0.21 & 0.11 & 0.5 & 0.46 & 2.7 & 0.07 & 0.3 & 0.40 & 2.4 \\
\hline B1-MM3 ${ }^{a}$ & $1800 \times 1300$ & 12 & 0.16 & 0.21 & 0.13 & 0.6 & 0.31 & 1.9 & 0.05 & 0.3 & 0.23 & 1.5 \\
\hline B1-MM4 ${ }^{a}$ & $4600 \times 3200$ & 12 & 0.21 & 0.21 & 0.13 & 0.6 & 0.78 & 3.7 & 0.06 & 0.3 & 0.61 & 2.9 \\
\hline B1B2-MM1 & $2700 \times 1800$ & 12 & 0.10 & 0.21 & 0.15 & 0.7 & 0.49 & 4.9 & 0.07 & 0.3 & 0.35 & 3.5 \\
\hline B2-MM1 & $<400$ & 12 & 0.14 & 0.21 & 0.15 & 0.7 & $<0.09$ & $<0.6$ & 0.05 & 0.3 & $<0.06$ & $<0.4$ \\
\hline B2-MM2 & $4500 \times 2400$ & 12 & 0.47 & 0.21 & 0.32 & 1.5 & 1.58 & 3.4 & & & & \\
\hline & & & & 0.21 & 0.08 & 0.4 & 0.55 & 1.2 & & & & \\
\hline B2-MM3 ${ }^{a}$ & $<400$ & 12 & 0.12 & 0.21 & 0.22 & 1.1 & $<0.12$ & $<1.0$ & & & & \\
\hline B2-MM4 & $2100 \times 960$ & 12 & 0.27 & 0.21 & 0.16 & 0.8 & 0.32 & 1.2 & 0.09 & 0.5 & 0.25 & 0.9 \\
\hline & & & & 0.21 & 0.13 & 0.7 & 0.29 & 1.1 & & & & \\
\hline B2-MM5 ${ }^{a}$ & $2200 \times 960$ & 12 & 0.26 & 0.21 & 0.20 & 1.0 & 0.41 & 1.6 & 0.12 & 0.6 & 0.27 & 1.1 \\
\hline B2-MM6 & $4300 \times 2700$ & 12 & 0.78 & 0.21 & 0.29 & 1.4 & 1.47 & 1.9 & 0.17 & 0.8 & 0.82 & 1.1 \\
\hline B2-MM7 & $<400$ & 12 & 0.23 & 0.21 & 0.33 & 1.6 & $<0.20$ & $<0.9$ & & & & \\
\hline B2-MM8 & $4000 \times 4000$ & 12 & 1.50 & 0.21 & 0.24 & 1.2 & 1.36 & 0.9 & 0.17 & 0.8 & 0.96 & 0.6 \\
\hline B2-MM9 & $1600 \times 960$ & 12 & 0.31 & 0.21 & 0.27 & 1.3 & 0.48 & 1.6 & & & & \\
\hline B2-MM10 & $3400 \times 2200$ & 12 & 0.60 & 0.21 & 0.23 & 1.1 & 0.90 & 1.5 & & & & \\
\hline B2-MM11 & $<400$ & 12 & 0.15 & 0.21 & 0.43 & 2.1 & $<0.31$ & $<2.0$ & 0.26 & 1.3 & $<0.15$ & $<1.0$ \\
\hline B2-MM12 & $2100 \times 1300$ & 12 & 0.39 & 0.21 & 0.23 & 1.1 & 0.52 & 1.3 & 0.08 & 0.4 & 0.28 & 0.7 \\
\hline B2-MM13 & $<400$ & 12 & 0.19 & 0.21 & 0.26 & 1.3 & $<0.15$ & $<0.8$ & & & & \\
\hline B2-MM14 & $2100 \times 1800$ & 12 & 0.43 & 0.21 & 0.32 & 1.6 & 0.96 & 2.2 & & & & \\
\hline B2-MM15 & $<400$ & 12 & 0.17 & 0.21 & 0.14 & 0.7 & $<0.08$ & $<0.5$ & 0.07 & 0.4 & $<0.06$ & $<0.4$ \\
\hline B2-MM16 & $2700 \times 1300$ & 12 & 0.35 & 0.21 & 0.26 & 1.3 & 0.70 & 2.0 & 0.17 & 0.8 & 0.45 & 1.3 \\
\hline B2-MM17 & $<400$ & 12 & 0.23 & 0.21 & 0.26 & 1.2 & $<0.15$ & $<0.6$ & 0.12 & 0.6 & $<0.08$ & $<0.3$ \\
\hline C-MM2 & $<400$ & 12 & 0.12 & 0.21 & 0.17 & 0.8 & $<0.10$ & $<0.8$ & & & & \\
\hline C-MM3 & $5400 \times 640$ & 12 & 0.23 & 0.21 & 0.12 & 0.6 & 0.35 & 1.5 & & & & \\
\hline C-MM4 & $2400 \times 1400$ & 12 & 0.16 & 0.21 & 0.13 & 0.6 & 0.37 & 2.3 & & & & \\
\hline C-MM5 & $<400$ & 12 & 0.10 & 0.21 & 0.12 & 0.6 & $<0.08$ & $<0.8$ & 0.08 & 0.4 & $<0.07$ & $<0.7$ \\
\hline C-MM6 & $4000 \times 3700$ & 12 & 0.33 & 0.21 & 0.13 & 0.6 & 0.76 & 2.3 & 0.09 & 0.4 & 0.65 & 2.0 \\
\hline C-MM7 & $<400$ & 12 & 0.13 & 0.21 & 0.11 & 0.5 & $<0.07$ & $<0.6$ & & & & \\
\hline E-MM2d & $4200 \times 2700$ & 12 & 0.63 & 0.21 & 0.11 & 0.5 & 0.61 & 1.0 & 0.09 & 0.4 & 0.58 & 0.9 \\
\hline E-MM4 & $6900 \times 5300$ & 12 & 0.61 & 0.21 & 0.13 & 0.6 & 1.20 & 2.0 & 0.09 & 0.4 & 1.04 & 1.7 \\
\hline F-MM1 & $4800 \times 2600$ & 12 & 0.35 & 0.21 & 0.19 & 0.9 & 0.95 & 2.7 & 0.13 & 0.6 & 0.70 & 2.0 \\
\hline F-MM $2^{a}$ & $2700 \times 1600$ & 12 & 0.17 & 0.21 & 0.14 & 0.7 & 0.43 & 2.6 & 0.12 & 0.6 & 0.39 & 2.3 \\
\hline
\end{tabular}

${ }^{a}$ Condensation with two velocity components in $\mathrm{N}_{2} \mathrm{H}^{+}$(see Table 2). Only the velocity component identified with Gaussclumps (see Table 3 ) is considered here.

${ }^{b}$ For some condensations, the dust temperatures adopted here to estimate $M_{1.2} \mathrm{~mm}$ from the measured $1.2 \mathrm{~mm}$ flux differ slightly from those assumed by MAN98. For simplicity, we used $T_{\mathrm{d}}=12 \mathrm{~K}$ for all condensations except SM1N, SM1, A-MM6, SM2, A-MM8 in Oph A, which are likely warmer $\left(T_{\mathrm{d}}=20 \mathrm{~K}\right.$, cf. André et al. 1993). The mass spectra shown in Figs. 1 and 8 were obtained using the dust temperatures quoted here.

MAN98). For unresolved condensations, the quoted $M_{\text {vir }}$ and $\alpha_{\text {vir }}$ values are only upper limits obtained under the assumption that the deconvolved $F W H M$ diameter is 400 AU (i.e., $\sim$ one fourth of the HPBW spatial resolution of the $1.2 \mathrm{~mm}$ continuum observations). Note also that our $\mathrm{N}_{2} \mathrm{H}^{+}(1-0)$ observations provide the line-of-sight velocity dispersion averaged over a $\sim 26^{\prime \prime}$ beam, while the FWHM angular size of the dust condensations ranges from $<15^{\prime \prime}$ to $\sim 30^{\prime \prime}$. However, since both $M_{\mathrm{vir}}$ and $M_{1.2} \mathrm{~mm}$ are derived for a diameter $\sim$ twice the $F W H M$ size, our method of estimating $\alpha_{\text {vir }}$ should be reasonably accurate. Some of the secondary components listed in Table 2 were ignored in Table 4, based on the Gaussclumps identifications summarized in Table 3.

The measured $\mathrm{N}_{2} \mathrm{H}^{+}(1-0)$ linewidths are narrow, indicative of small internal velocity dispersions. Prior to background subtraction, the average one-dimensional nonthermal velocity dispersion, $\sigma_{\mathrm{NT}}$, is estimated to be $\sim 0.20 \pm 0.08 \mathrm{~km} \mathrm{~s}^{-1}$ among the 43 components listed in Table 4 . More precisely, the average one-dimensional nonthermal velocity dispersion of the condensations ranges from $0.12 \pm 0.02 \mathrm{~km} \mathrm{~s}^{-1}$ in Oph E, $0.13 \pm$ $0.02 \mathrm{~km} \mathrm{~s}^{-1}$ in Oph C, $0.15 \pm 0.04 \mathrm{~km} \mathrm{~s}^{-1}$ in Oph B1/B1B2, $0.17 \pm 0.04 \mathrm{~km} \mathrm{~s}^{-1}$ in Oph F, to $0.20 \pm 0.08 \mathrm{~km} \mathrm{~s}^{-1}$ in Oph A, 
and $0.24 \pm 0.08 \mathrm{~km} \mathrm{~s}^{-1}$ in Oph B2. For comparison, the isothermal sound speed or one-dimensional thermal velocity dispersion is $\sigma_{\mathrm{T}} \sim 0.21-0.27 \mathrm{~km} \mathrm{~s}^{-1}$ for gas temperatures of $12-20 \mathrm{~K}$. Therefore, the condensations of Oph A, Oph B1, Oph C, Oph E, and Oph $\mathrm{F}$ are characterized by only subsonic levels of internal turbulence with $\sigma_{\mathrm{NT}} / \sigma_{\mathrm{T}} \sim 0.6-0.8$ on average, while the condensations of Oph B2 have at most "transonic" internal turbulence with $\sigma_{\mathrm{NT}} / \sigma_{\mathrm{T}} \sim 1.1<2$ on average. This is especially true since the measured nonthermal component of the $\mathrm{N}_{2} \mathrm{H}^{+}(1-0)$ linewidth may overestimate the intrinsic level of internal turbulence in the presence of infall motions and/or rotation (see Sects. 4.1 and 4.2).

In contrast, the line-of-sight velocity dispersions measured on the scale of the parent $\mathrm{DCO}^{+}$cores are significantly larger (cf. Table 2 of Loren et al. 1990), with corresponding values of $\sigma_{\mathrm{NT}}$ ranging from $\sim 0.17 \mathrm{~km} \mathrm{~s}^{-1}$ in Oph E, $0.2-0.3 \mathrm{~km} \mathrm{~s}^{-1}$ in Oph C and Oph F, to $0.35-0.5 \mathrm{~km} \mathrm{~s}^{-1}$ in Oph A, Oph B1, and Oph B2. Furthermore, the values of $\sigma_{\mathrm{NT}}$ derived from $\mathrm{NH}_{3}$ observations of the cores tend to be larger, by up to a factor of 2 , than those inferred from $\mathrm{DCO}^{+}$(Loren et al. 1990; A. Wootten, private communication). For instance, $\sigma_{\mathrm{NT}}=0.5 \pm 0.1 \mathrm{~km} \mathrm{~s}^{-1}$ is estimated in Oph B1 from $\mathrm{NH}_{3}$ (Zeng et al. 1984), compared to $\sigma_{\mathrm{NT}}=0.4 \mathrm{~km} \mathrm{~s}^{-1}$ from $\mathrm{DCO}^{+}$. Thus, the internal velocity dispersions derived here for the compact condensations are typically a factor of $\sim 2$ smaller than the nonthermal velocity dispersions of their parent dense cores. We conclude that the level of turbulence decreases from marginally supersonic on the scale of the $\mathrm{DCO}^{+}$cores to transonic or marginally subsonic on the scale of the prestellar condensations.

The above results are reminiscent of the transition to "coherence" observed by Goodman et al. (1998) in isolated starless cores. They suggest that, at least in nearby cluster-forming clouds, the initial conditions for individual protostellar collapse are "coherent" and largely free of turbulence. They are also consistent with scenarios, such as the "kernel" model of Myers (1998), according to which protocluster condensations form by dissipation of MHD turbulence on small scales within massive, turbulent cores (see also Nakano 1998).

\section{Motions within the $\mathrm{DCO}^{+}$cores of $\mathrm{L} 1688$}

\subsection{Evidence of gravitational infall motions}

Our data show the classical spectroscopic signature of infall motions (cf. Evans 1999; Myers et al. 2000) toward at least six $1.2 \mathrm{~mm}$ continuum condensations, where optically thick lines such as $\mathrm{CS}(2-1), \mathrm{CS}(3-2), \mathrm{H}_{2} \mathrm{CO}\left(2_{12}-1_{11}\right)$, and/or $\mathrm{HCO}^{+}(3-2)$ are double-peaked with a stronger blue peak, while low-opticaldepth lines such as $\mathrm{N}_{2} \mathrm{H}^{+}(101-012)$ and $\mathrm{C}^{34} \mathrm{~S}(2-1)$ peak in the dip between the blue and red peaks of the optically thick tracers (cf. Fig. 5) ${ }^{4}$. Such asymmetric line profiles skewed to the blue in optically thick tracers are produced when a gradient in excitation temperature toward source center is combined with inward motions (e.g. Leung \& Brown 1977). Here, among a total of 25 starless condensations observed in at least one optically thick infall tracer and one optically thin transition, blue infall profiles are clearly observed in 6 condensations (A-SM2, B2-MM16, CMM5, C-MM6, E-MM2d, and E-MM4, see Fig. 5), tentatively observed in 10 other condensations (A-MM4, A-MM5, A-SM1,

4 The optically thick spectra observed toward C-MM5 and C-MM6 show two fainter, additional peaks at velocities $\sim 2.8$ and $\sim 4.9 \mathrm{~km} \mathrm{~s}^{-1}$. These additional velocity components are also observed in $\mathrm{C}^{18} \mathrm{O}(2-1)$ but are not seen in $\mathrm{N}_{2} \mathrm{H}^{+}(1-0)$. They likely arise from the ambient cloud rather than from C-MM5 et C-MM6 themselves.

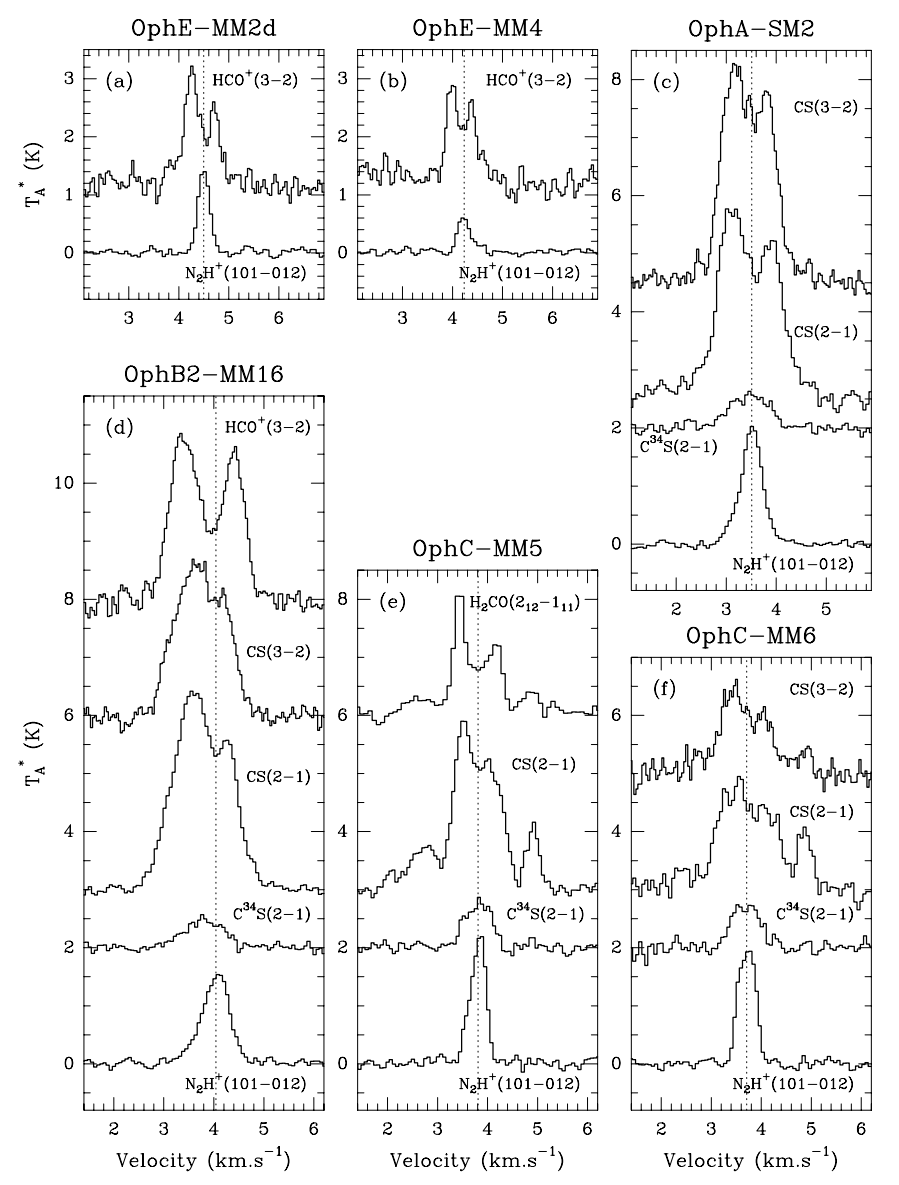

Fig. 5. Spectroscopic signatures of infall motions observed toward 6 Ophiuchus starless condensations: a) OphE-MM2d, b) OphE-MM4, c) OphA-SM2, d) OphB2-MM16, e) OphC-MM5, and f) OphCMM6. On each panel, the vertical dotted line marks the source systemic velocity as derived from a Gaussian HFS fit to the observed $\mathrm{N}_{2} \mathrm{H}^{+}(1-0)$ spectrum.

A-MM8, C-N, B2-MM12, B2-MM13, B2-MM14, B2-MM15, B2-MM17), and not seen in the remaining 9 sources (A-MM6, A-SM1N, F-MM1, F-MM2, B1-MM4, B2-MM2, B2-MM6, B2MM8, B2-MM10).

In the case of A-SM2 and C-MM6, the absorption dip observed in the optically thick line profiles is slightly redshifted with respect to the LSR velocity traced by the optically thin lines, by $\sim 0.1$ and $\sim 0.1-0.2 \mathrm{~km} \mathrm{~s}^{-1}$, respectively. This suggests that the outer gas layers responsible for the absorption dip in these two condensations are characterized by significant inward velocities $\sim 0.1 \mathrm{~km} \mathrm{~s}^{-1}$ (see, e.g., discussion in Sect. 3.3 of Belloche et al. 2002). By contrast, the absorption dip is not redshifted (nor blueshifted) toward E-MM2d, E-MM4, B2-MM16, and C-MM5, suggesting that only comparatively deeper layers undergo infall motions in the latter sources.

\subsection{Large-scale velocity structure}

In order to investigate the presence of systematic velocity gradients, such as rotational gradients, within the $\mathrm{DCO}^{+}$cores of L1688, we plot maps of the $\mathrm{N}_{2} \mathrm{H}^{+}(1-0)$ centroid velocity across Oph A, Oph B1, Oph B2, Oph C-S, Oph E, and Oph F in Figs. $6 \mathrm{a}-\mathrm{f}$. These centroid velocity maps were derived from the original $\mathrm{N}_{2} \mathrm{H}^{+}(1-0)$ data cubes before background subtraction. No clear, large-scale velocity gradient can be seen in the velocity 


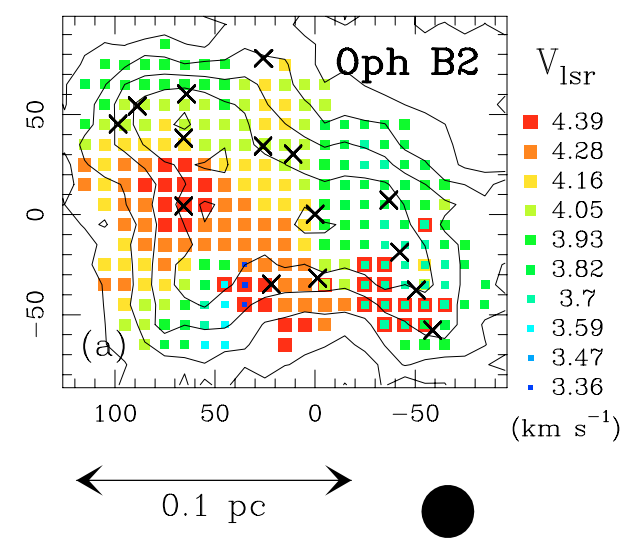

Beam

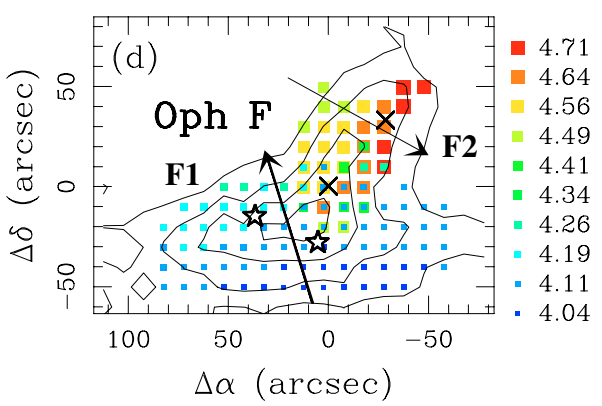

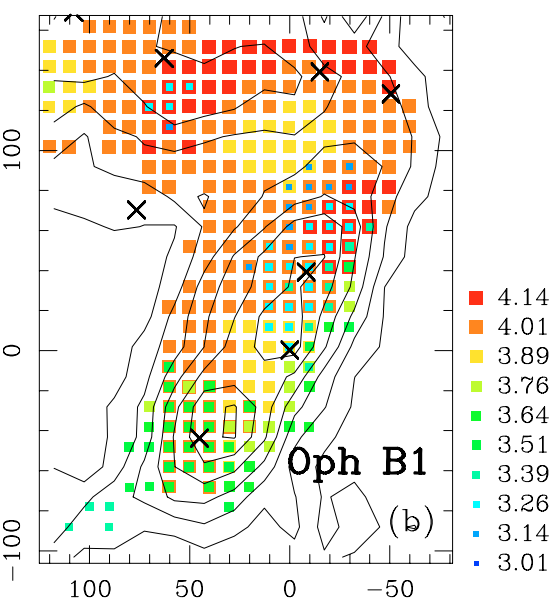
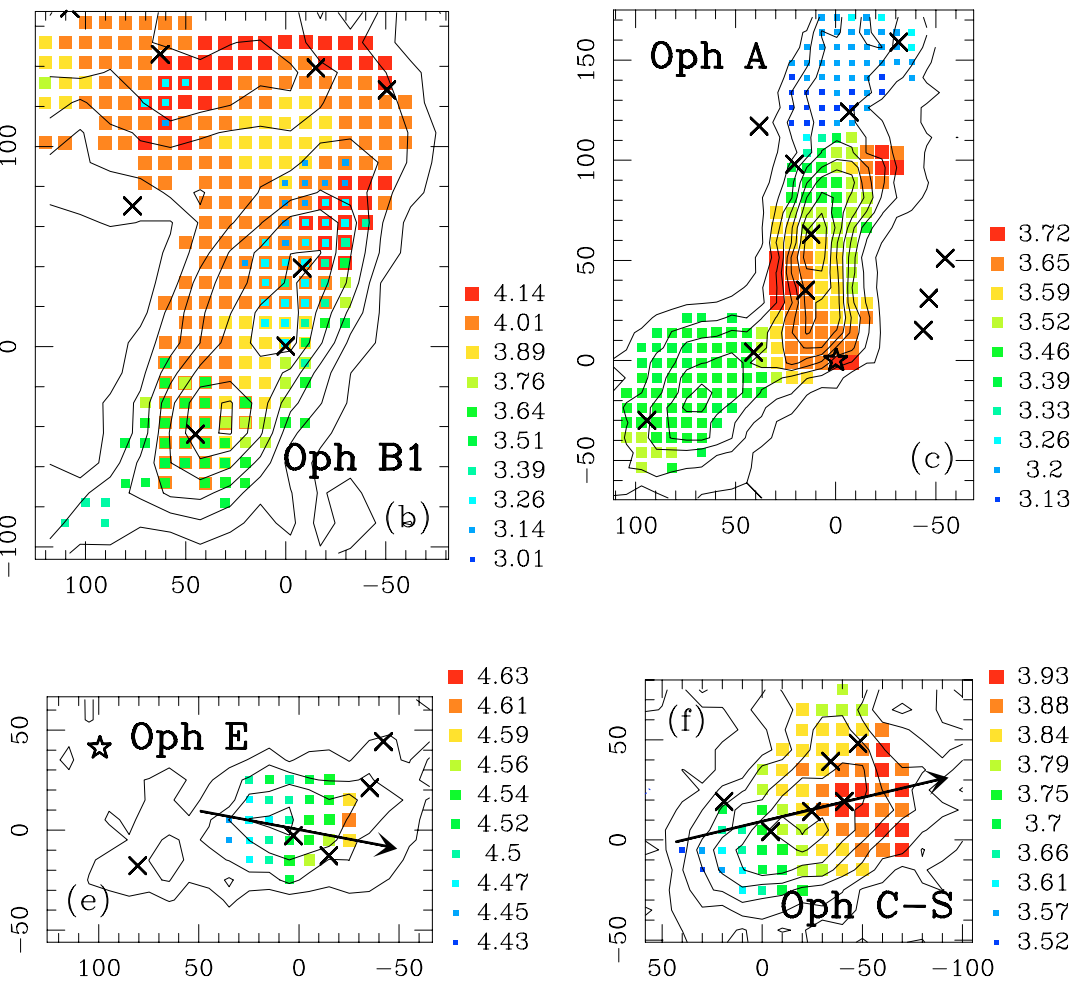

Fig. 6. Centroid velocity maps of a) Oph B2, b) Oph B1, c) Oph A, d) Oph F, e) Oph E, and f) Oph C-S as derived from HFS fits with one or two velocity components to the $\mathrm{N}_{2} \mathrm{H}^{+}(1-0)$ spectra with a signal-to-noise ratio larger than $\sim 5$ (filled squares of varying sizes and colors). The (0, 0) positions are given in the caption of Fig. 2. The linear size of the squares increases, and their color changes from blue to red, as $V_{\text {lsr }}$ increases. The underlying contours represent the same $\mathrm{N}_{2} \mathrm{H}^{+}(1-0)$ integrated intensity maps as shown in Figs. 2a-f. The direction of the fitted velocity gradients in Oph F, Oph C-S, and Oph E are shown by arrows in panels d), e), and f) (see Table 5). In Oph F, two velocity components were fitted separately and the arrows show the two corresponding velocity gradients associated with components Oph F1 at $v \sim 4.2 \mathrm{~km} \mathrm{~s}^{-1}$ and Oph F2 at $v \sim 4.6 \mathrm{~km} \mathrm{~s}^{-1}$, respectively. Crosses mark the $1.2 \mathrm{~mm}$ continuum positions of starless condensations (MAN98), while stars mark the positions of protostars (VLA 1623, YLW 15/IRS 43, CRBR 85, and WL 19).

maps of Oph A, Oph B1, and Oph B2, and the underlying kinematic structure cannot be described as a simple superposition of a small number of velocity gradients. In Oph A, which resembles a curved filament in the integrated intensity map, the two ends of the filament are characterized by lower systemic velocities $\left(V_{\mathrm{lsr}} \sim 3.2\right.$ and $\left.\sim 3.5 \mathrm{~km} \mathrm{~s}^{-1}\right)$ than the central region $\left(V_{\mathrm{lsr}} \sim 3.7 \mathrm{~km} \mathrm{~s}^{-1}\right)$, dominated by the objects A-SM1 and VLA 1623. In Oph B2, the two condensations B2-MM10 and B2-MM15 stand out with significantly higher systemic velocities $\left(V_{\mathrm{lsr}} \sim 4.4 \mathrm{~km} \mathrm{~s}^{-1}\right)$ than the bulk of the core $\left(V_{\mathrm{lsr}} \sim\right.$ $3.7-4.1 \mathrm{~km} \mathrm{~s}^{-1}$ ). In Oph B1, two velocity components are detected on the lines of sight to B1-MM2, B1-MM3, and B1-MM4. Based on the results of our Gaussclumps analysis (see Table 3), we believe that only the $\sim 4.0 \mathrm{~km} \mathrm{~s}^{-1}$ component, connected to Oph B2, is physically associated with the compact condensations B1-MM2, B1-MM3, and B1-MM4.

Well-defined $\mathrm{N}_{2} \mathrm{H}^{+}(1-0)$ velocity gradients were identified toward Oph C-S, Oph E, and Oph F, and their amplitudes estimated using the least-squares fitting method of Goodman et al. (1993). In particular, Oph C-S is characterized by a systematic projected velocity gradient of mean value $\|\nabla V\| \sim$ $4.4 \mathrm{~km} \mathrm{~s}^{-1} \mathrm{pc}^{-1}$, with velocities increasing along the direction of position angle $\mathrm{PA} \sim-77^{\circ}$ in the plane of the sky (see Table 5 and Fig. 6f).

In Oph E, our $\mathrm{N}_{2} \mathrm{H}^{+}(1-0)$ data suggest the presence of a velocity gradient $\|\nabla V\| \sim 3.0 \mathrm{~km} \mathrm{~s}^{-1} \mathrm{pc}^{-1}$ at $\mathrm{PA} \sim-101^{\circ}$ (cf. Fig. 6e). The signal-to-noise ratio in our $\mathrm{N}_{2} \mathrm{H}^{+}(1-0)$ map is however insufficient to constrain the nature of the velocity field.
Fortunately, we can also use our higher signal-to-noise maps taken in $\mathrm{H}^{13} \mathrm{CO}^{+}(1-0), \mathrm{DCO}^{+}(2-1)$, and $\mathrm{DCO}^{+}(3-2)$ to further constrain the velocity structure. For Oph $\mathrm{E}$ as a whole, the three tracers indicate a mean velocity gradient $5.2 \pm 0.3 \mathrm{~km} \mathrm{~s}^{-1} \mathrm{pc}^{-1}$ at $\mathrm{PA}=-108^{\circ} \pm 4^{\circ}$, in fairly good agreement with the gradient suggested by the $\mathrm{N}_{2} \mathrm{H}^{+}(1-0)$ data (cf. Fig. 6e).

In Oph $\mathrm{F}$, our $\mathrm{N}_{2} \mathrm{H}^{+}(1-0)$ data indicate the presence of two distinct velocity components, overlapping near the position of F-MM2 in the plane of the sky (see Fig. 6d). Oph F may thus actually consist of two independent dense cores, Oph F1 and Oph F2, with differing line-of-sight velocities ( 4.2 and $\sim 4.6 \mathrm{~km} \mathrm{~s}^{-1}$ ). Figure $6 \mathrm{~d}$ shows the direction of the mean velocity gradient derived in each of these two cores (see Table 5 for derived parameters). The fact that the velocity gradient found in Oph F1 $\left(\sim 3.8 \mathrm{~km} \mathrm{~s}^{-1} \mathrm{pc}^{-1}\right.$ at PA $\left.\sim 17^{\circ}\right)$ is nearly opposite to the gradient in Oph F2 $\left(\sim 6.3 \mathrm{~km} \mathrm{~s}^{-1} \mathrm{pc}^{-1} \text { at PA } \sim-119^{\circ}\right)^{5}$ supports the view that Oph $\mathrm{F}$ is made up of two independent cores, partly overlapping in projection, and not a single core with a mean velocity gradient $\sim 1.8 \mathrm{~km} \mathrm{~s}^{-1} \mathrm{pc}^{-1}$ at $\mathrm{PA} \sim-50^{\circ}$ as suggested by Loren et al. (1990) based on lower-resolution $\mathrm{DCO}^{+}(2-1)$ observations.

For L1688 considered as a whole (cf. Fig. 7), a global velocity gradient of $\sim 1.1 \mathrm{~km} \mathrm{~s}^{-1} \mathrm{pc}^{-1}$ is seen from North-West (Oph A) to South-East (Oph F) (see also Loren 1989 in ${ }^{13} \mathrm{CO}$ and Loren et al. 1990 in $\left.\mathrm{DCO}^{+}\right)$. The direction of this large-scale

5 The direction of the velocity gradient is however more uncertain in Oph F2 since it was derived using only 5 independent points. 
Table 5. Results of velocity gradient fitting.

\begin{tabular}{llcccccc}
\hline \hline Source & Transition & $\begin{array}{c}\text { Size }^{a} \\
\left(" \times \times^{\prime \prime}\right)\end{array}$ & $n_{\text {ind }}^{b}$ & $\begin{array}{c}V_{0}^{c} \\
\left(\mathrm{~km} \mathrm{~s}^{-1}\right)\end{array}$ & $\begin{array}{c}\|\nabla V\|^{c} \\
\left(\mathrm{~km} \mathrm{~s}^{-1} \mathrm{pc}^{-1}\right)\end{array}$ & $\begin{array}{c}\mathrm{PA}^{d} \\
\left({ }^{\circ}\right)\end{array}$ & $\begin{array}{c}\mathrm{rms}^{e} \\
\left(\mathrm{~km} \mathrm{~s}^{-1}\right)\end{array}$ \\
& $(2)$ & $(3)$ & $(4)$ & $(5)$ & $(6)$ & $(7)$ & $(8)$ \\
\hline Oph C-S & $\mathrm{N}_{2} \mathrm{H}^{+}(1-0)$ & $120 \times 70$ & 14 & 3.72 & 4.4 & -77 & 0.042 \\
\hline Oph F1 & $\mathrm{N}_{2} \mathrm{H}^{+}(1-0)$ & $150 \times 50$ & 16 & 4.17 & 3.8 & 17 & 0.026 \\
Oph F2 & $\mathrm{N}_{2} \mathrm{H}^{+}(1-0)$ & $50 \times 80$ & 5 & 4.60 & 6.3 & -119 & 0.028 \\
\hline Oph E & $\mathrm{H}^{13} \mathrm{CO}^{+}(1-0)$ & $180 \times 80$ & 19 & 4.47 & 5.0 & -106 & 0.052 \\
& $\mathrm{DCO}^{+}(2-1)$ & $170 \times 70$ & 43 & 4.53 & 5.1 & -104 & 0.051 \\
& $\mathrm{DCO}^{+}(3-2)$ & $160 \times 60$ & 68 & 4.48 & 5.6 & -113 & 0.044 \\
& Average & & & & $5.2(3)$ & $-108(4)$ & \\
\hline
\end{tabular}

Notes: the numbers in parentheses indicate the uncertainty in units of the last digit.

${ }^{a}$ Approximate diameter of the region over which a velocity gradient was fitted.

${ }^{b}$ Number of fully independent points used in the gradient fitting.

${ }^{c}$ The centroid velocity maps were fitted with the function $V_{0}+\boldsymbol{\nabla} V \cdot \Delta \boldsymbol{X}$, with $\boldsymbol{\Delta} \boldsymbol{X}$ the position vector measured from the $(0,0)$ position.

${ }^{d}$ Position angle of the direction of the velocity gradient $\nabla V$.

${ }^{e}$ rms residual of the fit.

gradient is $\mathrm{PA} \sim 120^{\circ}$ (cf. note $a$ of Table 6 ), as determined by a least-squares fit to the observed distribution of the line-of-sight systemic velocities of the condensations detected in $\mathrm{N}_{2} \mathrm{H}^{+}(1-0)$. The observed velocity pattern is however clearly more complex than that expected from simple large-scale rotation (see also Loren 1989). For instance, the direction of the mean velocity gradient changes to $\mathrm{PA} \sim 180^{\circ}$ if the condensations of Oph B1 and Oph B2 are ignored.

\subsection{Relative motions of the protocluster condensations within the $\mathrm{DCO}^{+}$cores}

Our estimates of the centroid line-of-sight velocities toward the prestellar condensations of L1688 (cf. Col. 7 of Table 2) provide interesting constraints on the relative motions between condensations. Figure 7 shows the distribution of systemic velocities as derived from our $\mathrm{N}_{2} \mathrm{H}^{+}(1-0)$ HFS fits, overlaid on the lowest contours of the $1.2 \mathrm{~mm}$ continuum map of MAN98.

Both the velocity differences between neighboring condensations and the overall velocity dispersion of the condensations within the L1688 protocluster are small. Table 6 gives the one-dimensional velocity dispersion, $\sigma_{1 \mathrm{D}}$, derived from our $\mathrm{N}_{2} \mathrm{H}^{+}(1-0)$ observations for various samples of condensations (cf. Cols. 6 and 7). This velocity dispersion was estimated in two ways, first around the mean LSR velocity of the condensations (estimate given in Col. 6), and second around the mean centroid $\mathrm{N}_{2} \mathrm{H}^{+}(101-012)$ velocity measured in the maps of Fig. 6 (estimate given in Col. 7). For the ensemble of 41 compact prestellar condensations and 3 protostars detected in $\mathrm{N}_{2} \mathrm{H}^{+}$, we estimate a global velocity dispersion $\sigma_{1 \mathrm{D}} \sim 0.36 \mathrm{~km} \mathrm{~s}^{-1}$ about the mean systemic velocity $\left\langle V_{\mathrm{lsr}}\right\rangle \sim 3.95 \mathrm{~km} \mathrm{~s}^{-1}$. Assuming isotropic random motions, this corresponds to a three-dimensional velocity dispersion $\sigma_{3 \mathrm{D}}=\sqrt{3} \sigma_{1 \mathrm{D}} \sim 0.62 \mathrm{~km} \mathrm{~s}^{-1}$ (cf. Cols. 8 and 9 of Table 6). If the velocity distribution is Maxwellian, the mean condensation speed relative to the center of mass of the system is $V_{\text {mean }}=\sqrt{8 / \pi} \sigma_{1 \mathrm{D}} \sim 0.57 \mathrm{~km} \mathrm{~s}^{-1}$ and the mean relative speed between condensations is $V_{\text {rel }}=\sqrt{2} V_{\text {mean }}=(4 / \sqrt{\pi}) \sigma_{1 \mathrm{D}} \sim$ $0.81 \mathrm{~km} \mathrm{~s}^{-1}$. Adopting a diameter $D \sim 1.1 \mathrm{pc}$ (e.g. Wilking \& Lada 1983) for the L1688 cloud, such a velocity dispersion implies a typical crossing time, $t_{\text {cross }} \equiv D / \sigma_{3 \mathrm{D}} \sim 1.8 \times 10^{6} \mathrm{yr}$ (cf. Cols. 10 and 11 of Table 6), for the condensations within the L1688 protocluster. After subtracting the global, systematic velocity gradient of $\sim 1.1 \mathrm{~km} \mathrm{~s}^{-1} \mathrm{pc}^{-1}$ seen across L1688 (cf. Sect. 4.2 above), the resulting 1D velocity dispersion of the condensations is even lower, $\sigma_{1 \mathrm{D}} \sim 0.25 \mathrm{~km} \mathrm{~s}^{-1}$, suggesting that the crossing time associated with purely random condensation motions is larger, $t_{\text {cross }} \sim 2.5 \times 10^{6} \mathrm{yr}$. The crossing times estimated separately within the individual $\mathrm{DCO}^{+}$cores with a statistically significant number of condensations (i.e., Oph A, B2, C) are only a factor of $\sim 2$ shorter, $t_{\text {cross }} \sim 0.6-1.0 \times 10^{6} \mathrm{yr}$.

\section{Discussion}

\subsection{Dynamical state and fate of the $L 1688$ condensations}

The starless condensations identified by MAN98 in L1688 are highly centrally concentrated and feature large density contrasts over the local background medium, strongly suggesting they are self-gravitating. More specifically, the estimated mean densities of the condensations exceed the mean densities of the parent $\mathrm{DCO}^{+}$cores by a typical factor $\sim 5-20$, while the mean column densities of the condensations exceed the background core column densities by a factor $\gtrsim 2$. For comparison, a critical selfgravitating Bonnor-Ebert isothermal spheroid has a mean density contrast $\bar{\rho}_{\mathrm{BE}} / \rho_{\mathrm{ext}} \sim 2.4$ (e.g. Lombardi \& Bertin 2001) and a mean column density contrast $\bar{\Sigma}_{\mathrm{BE}} / \Sigma_{\mathrm{ext}} \sim 1.5$ over the external medium.

The line observations reported in this paper provide more direct evidence that most of the L1688 condensations are gravitationally bound. Indeed, the narrow linewidths measured in $\mathrm{N}_{2} \mathrm{H}^{+}(1-0)$ (see Sect. 3.2) imply virial masses which generally agree within a factor of $\sim 2$ with the mass estimates derived by MAN98 from the $1.2 \mathrm{~mm}$ dust continuum. This can be considered a good agreement since both $M_{\text {vir }}$ and $M_{1.2} \mathrm{~mm}$ are themselves uncertain by a factor of $\sim 2$. On the theoretical side, self-gravitating condensations are expected to have virial mass ratios $\alpha_{\text {vir }} \equiv M_{\text {vir }} / M_{1.2 \mathrm{~mm}} \lesssim 2$, while objects in gravitational virial equilibrium should have $\alpha_{\text {vir }} \sim 1$ (e.g. Bertoldi \& McKee 1992). Here, among the 43 velocity components measured toward the 41 compact condensations positively detected in $\mathrm{N}_{2} \mathrm{H}^{+}(1-0), 15$ have an estimated virial mass ratio $\alpha_{\text {vir }}<1$ and another 23 have $\alpha_{\text {vir }} \lesssim 2.5$. Altogether, 38 components have $\alpha_{\text {vir }} \lesssim 2.5$ and only 5 have $\alpha_{\text {vir }} \gtrsim 3$. Among the latter 5 components, only 2 are associated with condensations more massive than $M_{1.2 \mathrm{~mm}} \sim 0.35 M_{\odot}$, of which one is associated with a condensation (B2-MM2) having another velocity component with $\alpha_{\text {vir }}<2$. Therefore, 37 of the 41 compact $1.2 \mathrm{~mm}$ condensations detected in $\mathrm{N}_{2} \mathrm{H}^{+}(1-0)$ have at least one velocity component with $\alpha_{\text {vir }} \lesssim 2.5$. 


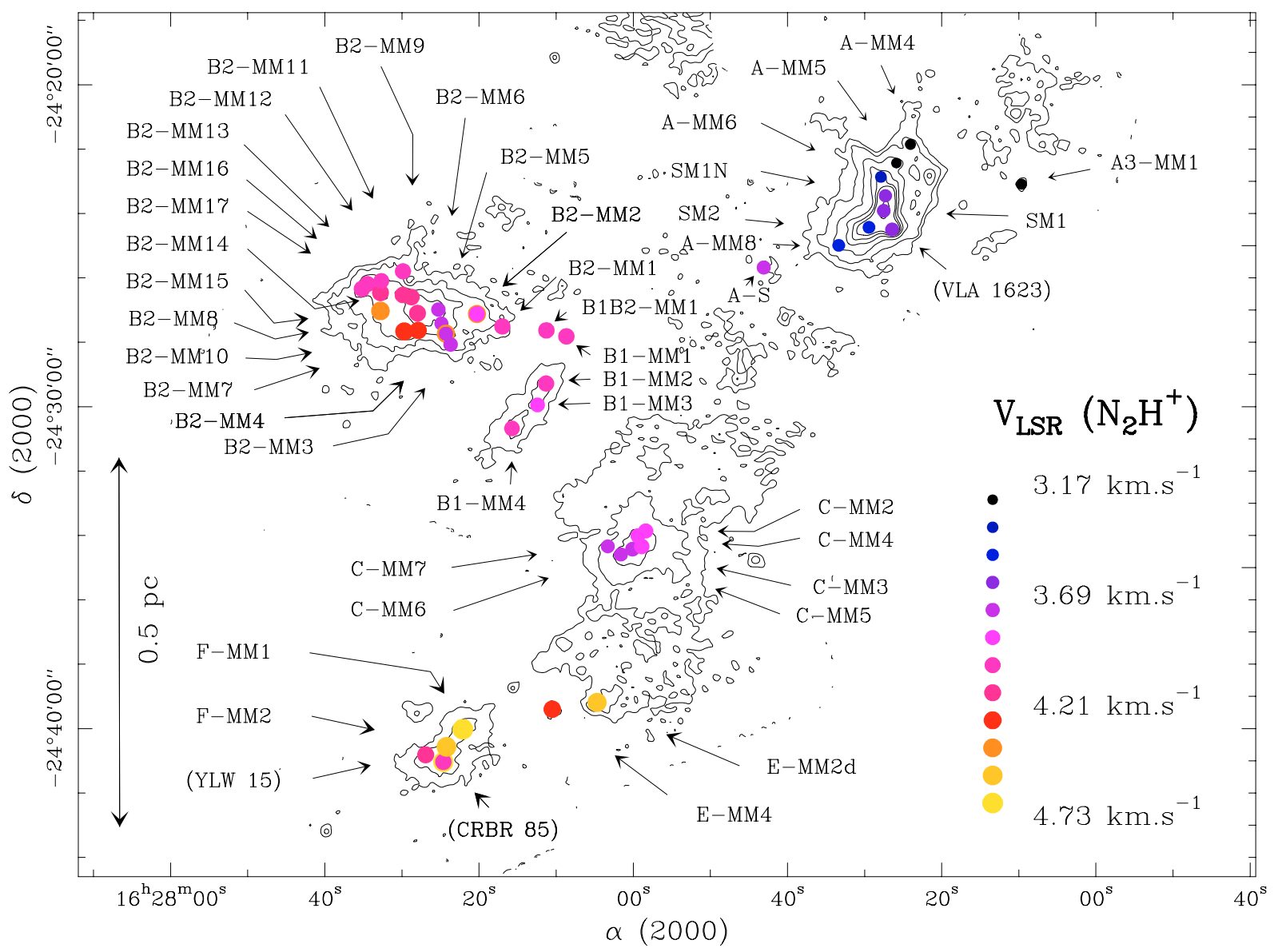

Fig. 7. Line-of-sight systemic velocities of the 41 prestellar condensations L1688 detected in $\mathrm{N}_{2} \mathrm{H}^{+}(1-0)$, overlaid on the lowest contours of the $1.2 \mathrm{~mm}$ continuum mosaic of MAN98. These velocities correspond to those listed in Col. [7] of Table 2. Each condensation is represented by a filled circle whose size increases with $V_{\mathrm{LSR}}$. The color coding varies from black to yellow with increasing Doppler shift. The systemic velocities of the protostars VLA 1623, YLW 15/IRS 43 and CRBR 85 are also shown. A few condensations have two markers, reflecting the presence of two velocity components in their spectra.

We conclude that a large majority $(\sim 75-90 \%)$ of the L1688 condensations detected in $\mathrm{N}_{2} \mathrm{H}^{+}(1-0)$, including $\sim 90 \%$ of those more massive than $\sim 0.35 M_{\odot}$, are likely gravitationally bound ${ }^{6}$. Coupled with the detection of signatures of infall motions toward some of them (see Sect. 4.1), this supports the notion that $75 \%$ of the starless condensations identified by MAN98 at $1.2 \mathrm{~mm}$ are prestellar in nature and on the verge of forming protostars (cf. André et al. 2000 and Ward-Thompson et al. 2007 for a definition of the prestellar stage). A fraction ( $30 \%)$ of the condensations with $M_{1.2 \mathrm{~mm}} \lesssim 0.35 M_{\odot}$ may be only marginally bound. The status of the condensations less massive than $\sim 0.1 M_{\odot}$ is less clear since these are weaker and often undetected in $\mathrm{N}_{2} \mathrm{H}^{+}$. Some of them may possibly correspond to unbound transient objects generated by supersonic turbulence (cf. Klessen et al. 2005). Interestingly, if a significant fraction of the condensations identified by MAN98 below $\sim 0.35 M_{\odot}$ are indeed unbound and not truly prestellar in nature, then the apparent excess of starless condensations at the low-mass end of the mass spectrum compared to the IMF of field systems (cf. Fig. 1) may find a natural explanation in the context of a one-to-one mapping between prestellar condensations and stellar systems.

${ }^{6}$ Note that the surface pressure term of the virial theorem, which we neglect here, tends to reduce the mass required for virial equilibrium, i.e., to increase the value of $\alpha_{\text {vir }}$ expected in virial equilibrium - see Bertoldi \& McKee (1992).

\subsection{Lifetime of the L1688 condensations}

The lifetime of the L1688 prestellar condensations is rather uncertain but several lines of reasoning suggest a typical value of $\sim 10^{5} \mathrm{yr}$. Table 7 provides estimates of some relevant evolutionary timescales. First, based on the measured mean densities $\bar{n}_{\mathrm{H} 2} \equiv \bar{\rho} / \mu m_{\mathrm{H}} \sim 10^{5}-10^{7} \mathrm{~cm}^{-3}$, the free-fall dynamical timescales of the condensations, $t_{\mathrm{ff}} \equiv(3 \pi / 32 G \bar{\rho})^{1 / 2}$, range from $t_{\mathrm{ff}} \sim 10^{4} \mathrm{yr}$ to $t_{\mathrm{ff}} \sim 10^{5} \mathrm{yr}$. While some of the condensations show evidence of infall motions, most of them are unlikely to be in free-fall collapse. We may conservatively estimate their evolutionary timescale to be $\sim 3 t_{\mathrm{ff}}$, which is appropriate for magnetically supercritical dense cores (e.g. Ciolek \& Mouschovias 1994) and typical of isolated prestellar cores detected in the submillimeter continuum (Kirk et al. 2005). This leads to timescale values ranging from $\sim 2 \times 10^{4} \mathrm{yr}$ to $\sim 3.5 \times 10^{5} \mathrm{yr}$ (cf. Col. 6 of Table 7). Second, assuming that the L1688 condensations evolve into stellar systems at a constant rate, we may derive a rough statistical estimate of their lifetime (Col. 7 of Table 7) by comparing the number of observed prestellar condensations (Col. 3 of Table 7) to the number of pre-main sequence (PMS) systems found in the same region (Col. 2 of Table 7). The ISOCAM mid-IR survey of Bontemps et al. (2001) revealed a total of 109 Class II PMS objects in L1688 with ages 0.4-1 Myr. If all of the 45 starless condensations identified by MAN98 above 
Table 6. Velocity dispersion of the L1688 protocluster condensations.

\begin{tabular}{lcccccccccc}
\hline \hline Sample $^{a}$ & $n^{b}$ & $\begin{array}{c}D^{c} \\
(\mathrm{pc})\end{array}$ & $\begin{array}{c}\left\langle V_{\mathrm{lsr}}^{d}\right\rangle \\
\left(\mathrm{km} \mathrm{s}^{-1}\right)\end{array}$ & $\begin{array}{c}\left\langle V_{\mathrm{cent}}^{e}\right\rangle \\
\left(\mathrm{km} \mathrm{s}^{-1}\right)\end{array}$ & $\begin{array}{c}\sigma_{1 \mathrm{D}}^{f} \\
\left(\mathrm{~km} \mathrm{~s}^{-1}\right)\end{array}$ & $\begin{array}{c}\sigma_{1 \mathrm{D}, \mathrm{c}}^{g} \\
\left(\mathrm{~km} \mathrm{~s}^{-1}\right)\end{array}$ & $\begin{array}{c}\sigma_{3 \mathrm{D}}^{h} \\
\left(\mathrm{~km} \mathrm{~s}^{-1}\right)\end{array}$ & $\begin{array}{c}\sigma_{3 \mathrm{D}, \mathrm{c}}^{i} \\
\left(\mathrm{~km} \mathrm{~s}^{-1}\right)\end{array}$ & $\begin{array}{c}D / \sigma_{3 \mathrm{D}} \\
\left(10^{6} \mathrm{yr}\right) \\
(10)\end{array}$ & $\begin{array}{c}D / \sigma_{3 \mathrm{D}, \mathrm{c}} \\
\left(10^{6} \mathrm{yr}\right) \\
(11)\end{array}$ \\
\hline Oph A & $(2)$ & $(3)$ & $(4)$ & $(5)$ & $(6)$ & $(7)$ & $(8)$ & $(9)$ & $(10)$ & 0.8 \\
Oph B1/B2 & 24 & 0.28 & 3.44 & 3.48 & $0.19(5)$ & $0.20(5)$ & $0.33(8)$ & $0.34(9)$ & 0.8 & 0.8 \\
Oph C,E,F & 10 & 0.44 & 4.05 & 3.96 & $0.20(3)$ & $0.22(3)$ & $0.35(5)$ & $0.38(6)$ & 1.0 & 0.9 \\
\hline L1688 & 47 & 1.10 & 3.95 & 3.05 & $0.39(9)$ & $0.39(9)$ & $0.67(16)$ & $0.68(16)$ & 0.7 & 0.6 \\
L1688 - $\nabla V$ & 47 & 1.10 & - & - & $0.36(4)$ & $0.36(4)$ & $0.62(7)$ & $0.63(7)$ & 1.8 & 1.7 \\
\hline
\end{tabular}

Notes: the numbers in parentheses indicate the uncertainty in units of the last digit.

${ }^{a}$ The first three samples contain the velocity components of Table 4 only. The last two samples include the protostars in addition. For the "L1688 $-\nabla V$ " sample, the velocity dispersion is computed after removing the large scale velocity gradient measured with the method described in note $c$ of Table 5 using $\mathrm{C}-\mathrm{N}$ as the $(0,0)$ position: $V_{0}=3.87 \mathrm{~km} \mathrm{~s}^{-1},\|\nabla V\|=1.1 \mathrm{~km} \mathrm{~s}^{-1} \mathrm{pc}^{-1}, \mathrm{PA}=117^{\circ}$.

${ }^{b}$ Number of velocity components used for the calculations.

${ }^{c}$ Diameter of the region containing each sample.

${ }^{d}$ Mean LSR velocity of the components, computed with the velocities listed in Col. 5 of Table 2.

${ }^{e}$ Mean centroid velocity computed on the $\mathrm{N}_{2} \mathrm{H}^{+}(101-012)$ spectra shown in Fig. 6.

${ }^{f}$ Standard deviation of the distribution of component LSR velocities around $\left\langle V_{\mathrm{lsr}}\right\rangle$ given in Col. 4. The error bar was estimated as $\sigma_{1 \mathrm{D}} / \sqrt{2(n-1)}$, assuming that the sample is drawn from a larger population whose velocity distribution follows Gaussian statistics.

${ }^{g}$ Same as in Col. 6 but computed around $\left\langle V_{\text {cent }}\right\rangle$ given in Col. 5.

${ }^{h}$ 3D velocity dispersion calculated from $\sigma_{1 \mathrm{D}}$ assuming isotropic motions. The error bar was scaled from that estimated for $\sigma_{1 \mathrm{D}}$.

${ }^{i}$ Same as in Col. 8 but computed around $\left\langle V_{\text {cent }}\right\rangle$ given in Col. 5 .

Table 7. Evolutionary timescales for various systems of protocluster condensations.

\begin{tabular}{lcccccccc}
\hline \hline Sub-cluster & $\begin{array}{c}\text { Nb of } \\
\text { Class II } \\
\text { YSOs }\end{array}$ & $\begin{array}{c}\text { Nb of starless } \\
\text { condensations } \\
>0.1 M_{\odot}\end{array}$ & $1+\Theta$ & $\frac{R^{2}}{N_{\text {cond }}^{2} r_{\text {cond }}}$ & $3 t_{\text {ff }}$ & $\begin{array}{c}\text { Statistical } \\
\text { lifetime }\end{array}$ & $t_{\text {cross }}$ & $t_{\text {coll }}$ \\
& 109 & 45 & 2 & 35 & $0.2-3.5$ & $2-4$ & 18 & 160 \\
$\left(10^{5}\right.$ yr $)$ & $\left(10^{5}\right.$ yr $)$ & $\left(10^{5}\right.$ yr $)$ \\
\hline L1688 & 41 & 9 & 8 & 10 & $0.5-2$ & $0.9-2$ & 8 & 5.5 \\
Oph A & 31 & 22 & 3.5 & 15 & $0.2-2.5$ & $3-7$ & 10 & 22 \\
Oph B1/B2 & 35 & 12 & 3 & 15 & $0.3-3.5$ & $2-4$ & 7 & 19 \\
Oph C/E/F & 35 & & & & & & & \\
\hline
\end{tabular}

$0.1 M_{\odot}$ are truly prestellar in nature, this points to a prestellar lifetime $\sim 2-4 \times 10^{5}$ yr. A third timescale estimate may be obtained by dividing the typical condensation outer radius $r_{\text {cond }} \sim 4000 \mathrm{AU}$ by a typical infall speed $V_{\text {inf }} \sim 0.1-0.3 \mathrm{~km} \mathrm{~s}^{-1}$ (cf. Belloche et al. 2001). This approach yields a condensation lifetime $\sim 0.6-2 \times 10^{5} \mathrm{yr}$. We conclude that the $1.2 \mathrm{~mm}$ continuum condensations of L1688 are likely characterized by a range of lifetimes between $\sim 2 \times 10^{4} \mathrm{yr}$ and $\sim 5 \times 10^{5} \mathrm{yr}$ (see Cols. 6 and 7 of Table 7).

As pointed out by Clark et al. (2007), if the lifetime of the condensations depends on their mass, then the observed mass spectrum is not necessarily representative of the intrinsic condensation mass distribution (CMD) (see also Elmegreen 2000). This is due to the fact that an observer is more likely to detect long-lived condensations than short-lived condensations. Here, however, the mean densities of the L1688 condensations are essentially uncorrelated with their masses, so that there is no systematic dependence of the dynamical timescale on the mass. To quantify the importance of the potential timescale bias, we plot, in Fig. 8, a weighted version of the central Ophiuchus CMD in which each condensation was assigned a weight equal to $\frac{\left\langle t_{\mathrm{ff}}\right\rangle}{t_{\mathrm{ff}}}=\frac{\bar{\rho}^{1 / 2}}{\left\langle\bar{\rho}^{1 / 2}\right\rangle}$ (instead of 1 as used for Fig. 1), where $\left\langle t_{\mathrm{ff}}\right\rangle$ is the average free-fall time of the condensations. Such a weighting should allow us to recover the intrinsic shape of the CMD assuming that the lifetime of each condensation is proportional to its free-fall time. As can be seen in Fig. 8, this weighting does not change the high-mass end of the CMD and only affects the low-mass end. We conclude that the steep, Salpeter-like slope of

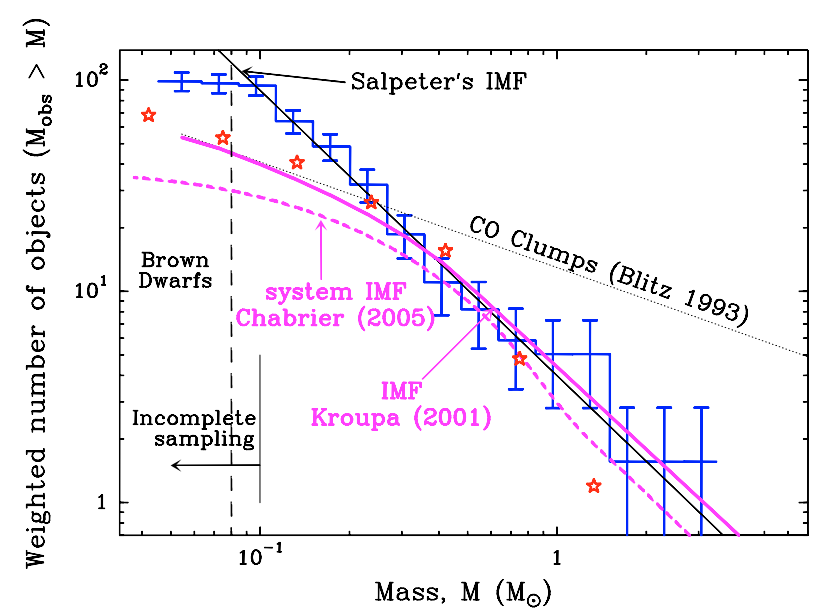

Fig. 8. Weighted cumulative mass spectrum of the 57 starless condensations identified by MAN98 (histogram with error bars), compared to the same mass distributions as shown in Fig. 1, as well as the Salpeter power-law IMF (solid line). Here, each condensation was assigned a weight inversely proportional to its estimated free-fall dynamical timescale (see text). The flattening apparent below $\sim 0.4 M_{\odot}$ in the unweighted CMD (Fig. 1) is not seen in the weighted CMD shown here, which is essentially consistent with a single, Salpeter-like power law.

the CMD at the high-mass end is robust, but that the presence of a break at $\sim 0.4 M_{\odot}$ is less robust. 


\subsection{Likelihood of interactions between condensations}

Using the velocity dispersions derived for the condensations in Sect. 4.3 and the lifetime estimates of Sect. 5.2 above, we are now in a position to assess whether dynamical interactions between condensations are a frequent or rare phenomenon in the L1688 protocluster.

Assuming an isotropic, Maxwellian velocity distribution, the time, $t_{\text {coll }}$, required for a condensation to interact or collide with another condensation can be estimated from the following formula for the collision rate $1 / t_{\text {coll }}$ :

$1 / t_{\text {coll }}=4 \sqrt{\pi} \times n_{\text {cond }} \sigma_{1 \mathrm{D}} r_{\text {cond }}^{2} \times(1+\Theta)$,

where $n_{\text {cond }}$ is the number density of condensations, $r_{\text {cond }}$ is the condensation outer radius (which we take to be twice the measured HWHM radius at $1.2 \mathrm{~mm}-$ cf. Sect. 3.2), $\pi r_{\text {cond }}^{2}$ is the collisional cross section of the condensation, and $1+\Theta \equiv$ $1+G M_{\text {cond }} /\left(\sigma_{1 \mathrm{D}}^{2} r_{\text {cond }}\right)$ is the gravitational focusing factor (e.g. Binney \& Tremaine 1987). Further assuming that the condensations are homogeneously distributed in a spherical system of radius $R$, so that $n_{\text {cond }}=N_{\text {cond }} /\left(\frac{4}{3} \pi R^{3}\right)$, the ratio of $t_{\text {coll }}$ to $t_{\text {cross }}$ takes on the simple form:

$\frac{t_{\text {coll }}}{t_{\text {cross }}}=\frac{1}{2} \sqrt{\frac{\pi}{3}} \times \frac{R^{2}}{N_{\text {cond }} r_{\text {cond }}^{2}} \times \frac{1}{1+\Theta}$.

Using the above equation for the entire L1688 cluster-forming clump $\left(R \sim 0.55 \mathrm{pc}, N_{\text {cond }}=57, \sigma_{1 \mathrm{D}}=0.36 \mathrm{~km} \mathrm{~s}^{-1}\right)$ and adopting typical condensation properties $\left(r_{\text {cond }} \sim 2500 \mathrm{AU}\right.$ and $\left.M_{\text {cond }} \sim 0.4 M_{\odot}\right)$, we find $t_{\text {coll }}^{\mathrm{L} 1688} / t_{\text {cross }}^{\mathrm{L} 1688} \sim 9$ and thus $t_{\text {coll }}^{\mathrm{L} 1688} \sim$ $16 \times 10^{6} \mathrm{yr}$. The latter is two orders of magnitude larger than the estimated lifetime of the condensations (Sect. 5.2) and about one order of magnitude longer than the age of the L1688 PMS cluster (Bontemps et al. 2001). For simplicity, our analysis ignores the dynamical impact of the ambient protocluster environment. In such a dense environment, gas drag is likely to have an effect on the motions of individual condensations, which will make the associated velocity field depart from a true Maxwellian distribution. A detailed assessment of the effects of gas drag is beyond the scope of this paper, but qualitatively at least we expect the environment to make the interaction timescales even longer than the above estimates. We conclude that the interaction process is much too slow on the scale of the entire L1688 system to play any significant role in the evolution of the protocluster condensations.

Dynamical interactions are however more likely to occur on smaller scales than the whole L1688 cluster-forming clump. In their mid-IR census of the PMS cluster, Bontemps et al. (2001) identified three main sub-clusters associated with the $\mathrm{DCO}^{+}$ cores Oph A, Oph B1/B2, and Oph C/E/F, respectively. The fact that sub-clustering is observed in the spatial distribution of PMS objects further supports our conclusion that significant interactions between individual objects have not yet occurred on the scale of the entire protocluster. Using Eq. (2) for the condensations associated with each of the three sub-cluster systems $\left(R \sim 0.15 \mathrm{pc}, N_{\text {cond }}=10-20\right)$, we find shorter interaction timescales than for L1688 as a whole, $t_{\text {coll }}^{\text {sub }} \sim 6-22 \times 10^{5} \mathrm{yr}$ (see Col. 9 of Table 7). The derived collision timescales nevertheless remain significantly longer than the typical condensation lifetime $\sim 10^{5} \mathrm{yr}$, indicating that dynamical interactions between condensations cannot be a dominant process even on the scale of the sub-clusters and $\mathrm{DCO}^{+}$cloud cores. The collision timescales are even longer than the combined lifetime of the prestellar and protostellar (Class 0/Class I) phases, which we estimate to be at most $\sim 5 \times 10^{5} \mathrm{yr}$ (e.g. Greene et al. 1994). In general, therefore, the prestellar condensations do not have time to orbit through their parent $\mathrm{DCO}^{+}$core and collide with one another before evolving into Class II PMS objects. We note that $t_{\text {coll }}$ is only slightly larger than $5 \times 10^{5} \mathrm{yr}$ in the case of the Oph A cluster-forming core, suggesting that a few interactions may occur during the whole evolution of this sub-cluster. Interestingly, based on a recent detailed study of the NGC 2264-C clusterforming clump in the Mon OB1 complex, Peretto et al. (2006) found direct evidence of a strong dynamical interaction in the dense inner region of that protocluster. However, the interaction observed at the center of NGC 2264-C is purely gravitational in origin and results from large-scale, coherent collapse motions as opposed to random turbulent motions (Peretto et al. 2007).

Our present results in L1688 seem inconsistent with models which resort to strong dynamical interactions to build up a mass spectrum comparable to the IMF (e.g. Price \& Podsiadlowski 1995 - see also Sect. 5.4 below).

\subsection{Comparison with the competitive accretion picture}

In a scenario of clustered star formation proposed by Bonnell, Bate and collaborators (e.g. Bonnell et al. 2001a,b), the IMF is primarily determined by competitive accretion and dynamical interactions/ejections during the protostellar phase, corresponding observationally to Class 0 and Class I objects. In this picture, turbulence generates density fluctuations within molecular clouds, some of which are gravitationally unstable and collapse to interacting protostars or protostellar seeds. The initial envelope/core masses of these protostars are essentially uncorrelated with the corresponding final stellar masses, especially at the high-mass end of the IMF (cf. Bonnell et al. 2004). The protostars acquire most of their mass by moving around in the gravitational potential well of the parent cluster-forming clump and accreting background gas that initially did not belong to the corresponding protostellar envelopes/cores. This process of competitive accretion of background gas is highly non-uniform and depends primarily on the initial stellar position $R_{\star}$ within the protocluster. The few protostars initially located near the center of the cluster potential accrete rapidly from the start and become massive stars, while protostars in the low-density outer regions accrete much more slowly and become low-mass stars (see Bonnell et al. 2001b for a quantitative toy model consistent with the observed IMF).

In the context of this scenario, it is difficult to explain why the Ophiuchus prestellar condensations have a mass distribution resembling the IMF. Our present results on the kinematics of the protocluster condensations allow us to further discuss the possible relevance of the competitive accretion picture in the case of the L1688 embedded cluster. The rate at which a protostar accretes mass competitively as it travels through the background protocluster gas is $\dot{M}_{\text {acc }} \approx \pi \rho_{\text {back }} v_{\text {rel }} R_{\text {acc }}^{2}$ (cf. Bonnell et al. 2001a), which gives:

$$
\begin{gathered}
\dot{M}_{\mathrm{acc}} \sim 1.2 \times 10^{-6} M_{\odot} \mathrm{yr}^{-1}\left(\frac{n_{\mathrm{H}_{2}}^{\mathrm{back}}}{10^{5} \mathrm{~cm}^{-3}}\right) \\
\times\left(\frac{v_{\mathrm{rel}}}{0.3 \mathrm{~km} \mathrm{~s}^{-1}}\right)\left(\frac{R_{\mathrm{acc}}}{3000 \mathrm{AU}}\right)^{2}
\end{gathered}
$$

where $\rho_{\text {back }}$ is the background gas density, $v_{\text {rel }}$ is the relative velocity between the protostar and the local ambient gas, and $R_{\text {acc }}$ is the accretion radius. Bonnell et al. (2001a) have shown that a good analytic approximation for $R_{\text {acc }}$ is provided by the 
Table 8. Rates of competitive mass accretion for a range of objects in the L1688 protocluster.

\begin{tabular}{lcccc}
\hline \hline Object & $\begin{array}{c}n_{\mathrm{H}_{2}}^{\text {back }} \\
\left(\mathrm{cm}^{-3}\right)\end{array}$ & $\begin{array}{c}v_{\text {rel }} \\
\left(\mathrm{km} \mathrm{s}^{-1}\right)\end{array}$ & $\begin{array}{c}R_{\text {acc }} \\
(\mathrm{AU})\end{array}$ & $\begin{array}{c}\dot{M}_{\mathrm{acc}} \\
\left(M_{\odot} \mathrm{yr}^{-1}\right)\end{array}$ \\
\hline Class I in L1688 & $2 \times 10^{4}$ & 0.4 & 3500 & $4 \times 10^{-7}$ \\
Class I in DCO $^{+}$core & $5 \times 10^{4}$ & 0.3 & 3000 & $6 \times 10^{-7}$ \\
Class 0 in inner Oph A core & $4 \times 10^{5}$ & 0.3 & 2500 & $3 \times 10^{-6}$ \\
$\begin{array}{l}\text { Class 0 from multiple system } \\
\text { inside collapsing condensation }\end{array}$ & $10^{6}$ & 0.2 & 2000 & $3.5 \times 10^{-6}$ \\
Condensation in inner Oph A core & $4 \times 10^{5}$ & 0.3 & 2500 & $3 \times 10^{-6}$ \\
\hline
\end{tabular}

smaller of the Bondi-Hoyle radius, $R_{\mathrm{BH}}=2 G M_{\star} /\left(v_{\mathrm{rel}}^{2}+c_{\mathrm{s}}^{2}\right)$, and the local tidal-lobe radius, $R_{\text {tidal }} \approx 0.5\left(\frac{M_{\star}}{M_{\text {enc }}}\right)^{1 / 3} R_{\star}$, where $c_{\mathrm{s}}$ is the gas sound speed and $M_{\mathrm{enc}}\left(R_{\star}\right)$ is the mass enclosed within the protocluster at the protostar's position $R_{\star}$. The BondiHoyle radius, $R_{\mathrm{BH}}$, is the radius where the gravitational potential due to the protostar exceeds the kinetic energy of the gas. Here, we estimate the relative gas-protostar velocity to be the derived mean condensation speed $V_{\text {mean }}=\sqrt{8 / \pi} \sigma_{1 \mathrm{D}} \sim$ $0.3-0.4 \mathrm{~km} \mathrm{~s}^{-1} \lesssim 2 c_{\mathrm{s}}$, assuming a Maxwellian velocity distribution. Therefore, the typical value of the Bondi-Hoyle radius is: $R_{\mathrm{BH}} \sim 9900 \mathrm{AU}\left(M_{\star} / 0.5 M_{\odot}\right)\left(v_{\mathrm{rel}} / 0.3 \mathrm{~km} \mathrm{~s}^{-1}\right)^{-2}$.

The tidal radius, $R_{\text {tidal }}$, expresses the fact that the tidal forces exerted by the gravitational potential of the ambient protocluster limit the zone of influence of a given protostar. In gas-dominated protoclusters such as L1688, one usually has $R_{\text {tidal }} \lesssim R_{\mathrm{BH}}$ and thus $R_{\text {acc }} \approx R_{\text {tidal }}$; conversely, in stellar-dominated clusters $R_{\mathrm{BH}} \lesssim R_{\text {tidal }}$ and thus $R_{\mathrm{acc}} \approx R_{\mathrm{BH}}$ (Bonnell et al. 2001a).

To estimate the typical value of $R_{\text {tidal }}$ in the central Ophiuchus case, we assume that the overall gas distribution in the centrally-condensed L1688 protocluster and individual subclusters (e.g. Oph A) follows a $\rho \propto r^{-2}$ density profile on average. This assumption is consistent with the density gradient derived by MAN98 for the outer parts of the $\mathrm{DCO}^{+}$cores based on the circularly-averaged profiles observed at $1.2 \mathrm{~mm}$. Under this assumption, $M_{\mathrm{enc}}$ has a very simple expression, $M_{\mathrm{enc}}\left(R_{\star}\right)=$ $M_{\text {clus }} \times\left(R_{\star} / R_{\text {clus }}\right)$, and thus $R_{\text {tidal }} \approx 0.5\left(\frac{M_{\star}}{M_{\text {clus }}}\right)^{1 / 3} R_{\text {clus }}^{1 / 3} R_{\star}^{2 / 3}$, where $M_{\text {clus }}$ and $R_{\text {clus }}$ are the total (gas + stars) mass and outer radius of the protocluster, respectively. For the entire L1688 protocluster, we have $M_{\text {clus }} \sim 650 M_{\odot}, R_{\text {clus }} \sim 0.55 \mathrm{pc}$, and thus $R_{\text {tidal }}^{\mathrm{L} 1688} \sim$ $3500 \mathrm{AU}\left(M_{\star} / 0.5 M_{\odot}\right)^{1 / 3}\left(R_{\star} / 0.3 \mathrm{pc}\right)^{2 / 3}$. For the Oph A subcluster, we adopt $M_{\text {clus }} \sim 30 M_{\odot}, R_{\text {clus }} \sim 0.14 \mathrm{pc}$, and thus $R_{\text {tidal }}^{\text {OphA }} \sim 3000 \mathrm{AU}\left(M_{\star} / 0.5 M_{\odot}\right)^{1 / 3}\left(R_{\star} / 0.1 \mathrm{pc}\right)^{2 / 3}$. As expected, we see that $R_{\text {tidal }}<R_{\mathrm{BH}}$ and thus $R_{\text {acc }} \approx R_{\text {tidal }} \sim 3000 \mathrm{AU}$.

Based on these simple estimates, we find that the typical tidal-lobe radius of the L1688 condensations/protostars is comparable to the actual radius of the condensations and protostellar envelopes as measured by MAN98 at $1.2 \mathrm{~mm}$. This suggests that the overall gravitational potential of the L1688 protocluster does play an important role in limiting the condensation/envelope masses, in qualitative agreement with the competitive accretion picture (cf. Bonnell et al. 2001a, 2004). Note that the small value found here for the mean condensation velocity relative to the local gas $\left(V_{\text {mean }} \sim 0.3 \mathrm{~km} \mathrm{~s}^{-1}\right)$ is also consistent with the predictions of the competitive accretion model during the gas-dominated phase of protocluster evolution (Bonnell et al. 2001a). However, when we estimate the mass accretion rate resulting from competitive accretion in various typical situations, we find relatively low values (see Table 8), which are smaller than the infall rate expected from the gravitational collapse of individual condensations, i.e., $\dot{M}_{\mathrm{inf}} \sim 1-10 c_{\mathrm{s}}^{3} / G$ (see Shu 1977 and Foster \& Chevalier 1993 for model predictions, and Belloche et al. 2006 for an observed example). For a Class 0 protostar such as VLA 1623 embedded in the inner part of the Oph A $\mathrm{DCO}^{+}$core, we estimate the background gas density to be relatively high, $n_{\mathrm{H}_{2}}^{\text {back }} \sim 4 \times 10^{5} \mathrm{~cm}^{-3}$, and the tidal radius to be at most $R_{\text {tidal }}^{\mathrm{VLA} 1623} \sim 2500 \mathrm{AU}$, giving $\dot{M}_{\text {acc }} \sim 3 \times 10^{-6} M_{\odot} \mathrm{yr}^{-1}$. For comparison, the infall rate due to gravitational collapse is significantly higher at this early stage $\dot{M}_{\text {inf }} \gtrsim 10 c_{\mathrm{s}}^{3} / G \gtrsim 10^{-5}-10^{-4} M_{\odot} \mathrm{yr}^{-1}$ (Bontemps et al. 1996; Jayawardhana et al. 2001; André et al. 2001). For a more evolved Class I protostar such as YLW 15/IRS 43 embedded in the Oph $\mathrm{F} \mathrm{DCO}^{+}$core, the background gas density is lower, $n_{\mathrm{H}_{2}}^{\text {back }} \sim 5 \times 10^{4} \mathrm{~cm}^{-3}$, and the tidal radius is at most $R_{\text {tidal }}^{\mathrm{IRS} 43} \sim$ $3000 \mathrm{AU}$, giving $\dot{M}_{\mathrm{acc}} \sim 6 \times 10^{-7} M_{\odot} \mathrm{yr}^{-1}$. For comparison, the gravitational infall rate is $\dot{M}_{\mathrm{inf}} \sim c_{\mathrm{s}}^{3} / G \sim 2 \times 10^{-6} M_{\odot} \mathrm{yr}^{-1}$ at this late protostellar stage (e.g. Adams et al. 1987; Bontemps et al. 1996). For both Class 0 and Class I objects we thus find $\dot{M}_{\text {acc }}<\dot{M}_{\text {inf }} / 3$, implying that local gravitational collapse dominates over competitive accretion (see also Krumholz et al. 2005). We conclude that competitive accretion at the protostellar stage is unlikely to be the main mechanism responsible for determining the final masses of stellar systems in the central Ophiuchus star-forming cloud. If each prestellar condensation fragments into a small-N system during protostellar collapse (e.g. Goodwin et al. 2007), then competitive accretion may possibly play a more important role in defining the final masses of the individual components.

We also believe that competitive, Bondi-like accretion is more likely to operate at the prestellar stage and may possibly govern the growth of starless condensations within a cluster-forming cloud (cf. Bonnell et al. 2001b; Myers 2000; Basu \& Jones 2004). The growth rate of a typical $\sim 0.5 M_{\odot}$ condensation embedded in the inner part of a $\mathrm{DCO}^{+}$core such as Oph A should be similar to the competitive accretion rate estimated above for VLA 1623 , i.e., $\dot{M}_{\text {acc }} \sim 3 \times 10^{-6} M_{\odot} \mathrm{yr}^{-1}$. This is sufficient to approximately double the mass of the condensation in $\sim 2 \times 10^{5} \mathrm{yr}$, a timescale comparable to the condensation lifetime. However, once fast, nearly free-fall collapse sets in, we expect the growth rate to be quickly overwhelmed by the infall rate, so that a collapsing prestellar condensation should not have time to grow significantly in mass before evolving into a PMS system.

Another feature of the dynamics of a gas-dominated protocluster is that the entire system is expected to undergo global collapse/contraction, resulting in a centrally-condensed overall structure much like a self-gravitating isothermal sphere (cf. Adams 2000; Bonnell et al. 2001b). In this view, both the gas accretion and the protocluster evolution occur on the global dynamical timescale (see also Klessen \& Burkert 2000). 
Observationally, the velocity dispersion, $\sigma_{1 \mathrm{D}}$, estimated in Sect. 4.3 above for the L1688 condensations implies a binding virial mass $M_{\text {vir }} \approx 3 \times R \sigma_{1 \mathrm{D}}^{2} / G \sim 50 M_{\odot}$, which is much less than the total gas mass $\sim 550 M_{\odot}$ of the associated $\mathrm{C}^{18} \mathrm{O}$ cloud (cf. Wilking \& Lada 1983) and even less than the total stellar mass of the present infrared embedded cluster $\left(M_{\text {stars }} \sim 100 M_{\odot}\right.$, Bontemps et al. 2001). In other words, the observed condensation-to-condensation velocity dispersion is a factor of $\sim 3$ smaller than that expected in virial equilibrium (see also Table 6 of Peretto et al. 2006), Since the magnetic field does not seem to be strong enough to support the cloud (e.g. Troland et al. 1996), this comparison suggests that the L1688 system is indeed gravitationally unstable and possibly in an early state of large-scale, magnetically supercritical contraction. Interestingly, the $\mathrm{CO}$ and ${ }^{13} \mathrm{CO}$ lines observed toward the L1688 cloud exhibit the classical spectroscopic signature of contraction motions (cf. Sect. 4.1 above) over most of the protocluster extent (see Encrenaz et al. 1975). We also note that L1688 is not the only protocluster for which both subvirial relative speeds and evidence of large-scale contraction have been found. Two other examples are NGC 2264 (Peretto et al. 2006, 2007) and NGC 1333 (Walsh et al. 2006, 2007). This suggests that protoclusters often start their evolution from "cold", out-ofequilibrium initial conditions (cf. Adams et al. 2006), possibly as a result of the influence of external triggers (cf. Nutter et al. 2006).

\section{Summary and conclusions}

We carried out a detailed observational study of the kinematics of the L1688 protocluster condensations in the central Ophiuchus molecular cloud using $\mathrm{N}_{2} \mathrm{H}^{+}(1-0)$ as a tracer of dense gas. Additional observations were also taken in molecular lines such as $\mathrm{H}^{13} \mathrm{CO}^{+}(1-0), \mathrm{DCO}^{+}(2-1), \mathrm{HCO}^{+}(3-2)$, $\mathrm{CS}(2-1)$, and $\mathrm{C}^{34} \mathrm{~S}(2-1)$. Our main results and conclusions are as follows:

1. The $\mathrm{N}_{2} \mathrm{H}^{+}(1-0)$ line was positively detected toward 41 of the 57 compact starless condensations identified by MAN98 at $1.2 \mathrm{~mm}$, as well as 3 Class 0/Class I protostars (VLA 1623, CRBR 85, YLW 15/IRS 43). The same objects were also detected in $\mathrm{H}^{13} \mathrm{CO}^{+}(1-0)$ and/or $\mathrm{DCO}^{+}(2-1)$ when observed in these transitions. In addition, the Class I sources LFAM 26 and GY210 were detected in $\mathrm{H}^{13} \mathrm{CO}^{+}(1-0)$.

2. For 29 of the 44 condensations/protostars detected in $\mathrm{N}_{2} \mathrm{H}^{+}(1-0)$ we are confident that the line emission is at least partly associated with the compact $1.2 \mathrm{~mm}$ continuum object as opposed to the more extended parent cloud/ $\mathrm{DCO}^{+}$core, since the sources remained positively detected after subtraction of the local background line emission. Furthermore, at least 17 starless condensations and 3 protostars were found to have well-defined line counterparts in position-velocity space and could be identified in our background-subtracted $\mathrm{N}_{2} \mathrm{H}^{+}(101-012), \mathrm{H}^{13} \mathrm{CO}^{+}(1-0)$, or $\mathrm{DCO}^{+}(2-1)$ data cubes using the Gaussclumps clump-finding algorithm of Stutzki \& Güsten (1990).

3. The measured $\mathrm{N}_{2} \mathrm{H}^{+}(1-0)$ linewidths are narrow, indicative of small nonthermal velocity dispersions. The condensations of Oph A, Oph B1, Oph C, Oph E, and Oph F are characterized by subsonic levels of internal turbulence $\left(\sigma_{\mathrm{NT}} / \sigma_{\mathrm{T}}<1\right)$, while the condensations of Oph B2 have at most "transonic" internal turbulence $\left(\sigma_{\mathrm{NT}} / \sigma_{\mathrm{T}}<2\right)$.

4. We detected the classical spectroscopic signature of infall motions toward six $1.2 \mathrm{~mm}$ continuum condensations
(A-SM2, B2-MM16, C-MM5, C-MM6, E-MM2d, and EMM4). For these objects, the optically thick $\mathrm{CS}(2-1)$, $\mathrm{CS}(3-2), \mathrm{H}_{2} \mathrm{CO}\left(2_{12}-1_{11}\right)$, and/or $\mathrm{HCO}^{+}(3-2)$ lines are double-peaked with a stronger blue peak, while the lowoptical-depth $\mathrm{N}_{2} \mathrm{H}^{+}(101-012)$ line peaks in the dip of the optically thick line. The same signature was tentatively observed in 10 other condensations.

5. The virial masses derived from our $\mathrm{N}_{2} \mathrm{H}^{+}(1-0)$ detections of the condensations generally agree within a factor of $\sim 2$ with the masses estimated from the $1.2 \mathrm{~mm}$ dust continuum. On this basis, most ( $\sim 75-90 \%)$ of the L1688 condensations detected in $\mathrm{N}_{2} \mathrm{H}^{+}(1-0)$, including essentially all ( $\left.\sim 90 \%\right)$ of those more massive than $\sim 0.35 M_{\odot}$, appear to be gravitationally bound and prestellar in nature. The status of the starless $1.2 \mathrm{~mm}$ condensations less massive than $\sim 0.1 M_{\odot}$ is less clear since these were often undetected in $\mathrm{N}_{2} \mathrm{H}^{+}$.

6. We estimate that the prestellar condensations of L1688 are characterized by a range of lifetimes between $\sim 2 \times 10^{4} \mathrm{yr}$ and $\sim 5 \times 10^{5} \mathrm{yr}$. There is however no systematic dependence of lifetime on mass, so that the observed mass spectrum is not severely affected by the timescale bias pointed out by Clark et al. (2007). In particular, the steep, Salpeter-like slope of the condensation mass distribution at the high-mass end appears to be robust.

7. Based on the observed distribution of $\mathrm{N}_{2} \mathrm{H}^{+}(1-0)$ line centroid velocities, a global velocity dispersion $\sigma_{1 \mathrm{D}}<$ $0.4 \mathrm{~km} \mathrm{~s}^{-1}$ was estimated for the condensations of L1688. This condensation-to-condensation velocity dispersion is subvirial. It implies a crossing time $t_{\text {cross }} \sim 1$ Myr for the condensations within the parent protocluster and a typical collision time $t_{\text {coll }} \sim 1-10$ Myr between condensations. Since these timescales are longer than the estimated condensation lifetime $\sim 0.2-5 \times 10^{5} \mathrm{yr}$, we conclude that, in general, the L1688 prestellar condensations do not have time to orbit through their parent $\mathrm{DCO}^{+}$core and interact with one another before evolving into PMS objects.

8. Using an estimated mean relative velocity of $\sim 0.3-$ $0.4 \mathrm{~km} \mathrm{~s}^{-1}$ between protostellar envelopes and the background ambient gas, the mass accretion rate expected from competitive accretion was found to be significantly smaller than that resulting from gravitational collapse at the Class 0 stage. On the other hand, the typical tidal-lobe radius of the L1688 condensations/protostars was estimated to be comparable to the actual radius of the condensations and protostellar envelopes as measured in the dust continuum at $1.2 \mathrm{~mm}$. This suggests that the overall gravitational potential of the protocluster does play an important role in limiting the condensation/envelope masses, but that competitive accretion at the protostellar stage is not the dominant mechanism responsible for determining the final masses of stellar systems in the central Ophiuchus star-forming cloud.

9. We find that competitive, Bondi-like accretion is more likely to operate at the prestellar stage and propose that it may partly govern the growth of starless, self-gravitating condensations initially produced by gravoturbulent fragmentation toward an IMF, Salpeter-like mass spectrum.

Acknowledgements. We would like to thank Bruce Elmegreen and Ian Bonnell for enlightening discussions on protocluster dynamics. We are grateful to an anonymous referee for constructive comments that helped us improve the clarity of the paper.

\section{References}

Adams, F. C. 2000, ApJ, 542, 964 
Adams, F. C., \& Myers, P. C. 2001, ApJ, 553, 744

Adams, F. C., Lada, C. J., \& Shu, F. H. 1987, ApJ, 312, 788

Adams, F. C., Prozkow, E. M., Fatuzzo, M., \& Myers, P. C. 2006, ApJ, 641, 504

André, P., Ward-Thompson, D., \& Barsony, M. 1993, ApJ, 406, 122

André, P., Ward-Thompson, D., \& Barsony, M. 2000, Protostars and Planets IV, ed. V. Mannings, A. P. Boss, \& S. S. Russell (Tucson: University of Arizona Press), 59

André, P., Bouwman, J., Belloche, A., \& Hennebelle, P. 2003, in Chemistry as a Diagnostic of Star Formation, ed. C. L. Curry, \& M. Fich (NRC Press), 127

André, P., Motte, F., \& Belloche, A. 2001, in From Darkness to Light, ed. T. Montmerle, \& P. André, ASP Conf. Ser., 243, 209

Basu, S., \& Jones, C. E. 2004, MNRAS, 347, L47

Bate, M. R., Bonnell, I. A., \& Bromm, V. 2003, MNRAS, 339, 577

Belloche, A., André, P., \& Motte, F. 2001, in From Darkness to Light, ed. T. Montmerle, \& P. André, ASP Conf. Ser., 243, 313

Belloche, A., André, P., Despois, D., \& Blinder, S. 2002, A\&A, 393, 927

Belloche, A., Hennebelle, P., \& André, P. 2006, A\&A, 453, 145

Bertoldi, F., \& McKee, C. F. 1992, ApJ, 395, 140

Binney, J., \& Tremaine, S. 1987, Galactic dynamics, (Princeton, NJ: Princeton University Press), 1987

Blitz, L. 1993, in Protostars and Planets III, ed. E. H. Levy, \& J. I. Lunine (Tucson: Univ. of Arizona Press), 125

Bonnell, I., Bate, M., \& Zinnecker, H. 1998, MNRAS, 298, 93

Bonnell, I. A., Bate, M. R., Clarke, C. J., \& Pringle, J. E. 2001a, MNRAS, 323, 785

Bonnell, I. A., Clarke, C. J., Bate, M. R., \& Pringle, J. E. 2001b, MNRAS, 324, 573

Bontemps, S., André, P., Terebey, S., \& Cabrit, S. 1996, A\&A, 311, 858

Bonnell, I. A., Vine, S. G., \& Bate, M. R. 2004, MNRAS, 349, 735

Bontemps, S., André, P., Kaas, A. A., et al. 2001, A\&A, 372, 173

Brandner, W., Sheppard, S., Zinnecker, H., et al. 2000, A\&A, 364, L13

Caselli, P., Myers, P. C., \& Thaddeus, P. 1995, ApJ, 455, L77

Chabrier, G. 2003, PASP, 115, 763

Chabrier, G. 2005, ASSL 327: The Initial Mass Function 50 Years Later, ed. E. Corbelli, F. Palla, \& H. Zinnecker (Dordrecht: Springer), 41

Ciolek, G. E., \& Mouschovias, T. C. 1994, ApJ, 425, 142

Clark, P. C., Klessen, R. S., \& Bonnell, I. A. 2007, MNRAS, in press [arXiv:astro-ph/0704.2837]

Di Francesco, J., André, P., \& Myers, P. C. 2004, ApJ, 617, 425

Duchêne, G., Bouvier, J., Bontemps, S., André, P., \& Motte, F. 2004, A\&A, 427, 651

Elmegreen, B. G. 1997, ApJ, 486, 944

Elmegreen, B. G. 2000, in Star Formation from the Small to the Large Scale, ed. F. Favata, A. A. Kaas, \& A. Wilson, ESA SP-445, 265

Encrenaz, P. J., Falgarone, E., \& Lucas, R. 1975, A\&A, 44, 73

Evans, N. J. II 1999, ARA\&A, 37, 311

Foster, P. N., \& Chevalier, R. A. 1993, ApJ, 416, 303

Goodman, A. A., Benson, P. J., Fuller, G. A., \& Myers, P. C. 1993, ApJ, 406, 528 (GBFM93)

Goodman, A. A., Barranco, J. A., Wilner, D. J., \& Heyer, M. H. 1998, ApJ, 504, 223

Goodwin, S. P., Kroupa, P., Goodman, A., \& Burkert, A. 2007, Protostars and Planets V, ed. B. Reipurth, D. Jewitt, \& K. Keil (Tucson: University of Arizona Press), 133

Gottlieb, C. A., Myers, P. C., \& Thaddeus, P. 2003, ApJ, 588, 655

Greene, T. P., Wilking, B. A., André, P., Young, E. T., \& Lada, C. J. 1994, ApJ, 434,614
Jayawardhana, R., Hartmann, L., \& Calvet, N. 2001, ApJ, 548, 310

Johnstone, D., Wilson, C. D., Moriarty-Schieven, G., et al. 2000, ApJ, 545, 327

Kirk, J. M., Ward-Thompson, D., \& André, P. 2005, MNRAS, 360, 1506

Klessen, R. S., \& Burkert, A. 2000, ApJS, 128, 287

Klessen, R. S., Ballesteros-Paredes, J., Vázquez-Semadeni, E., \& Durán-Rojas, C. 2005, ApJ, 620, 786

Kramer, C., Stutzki, J., Rohrig, R., \& Corneliussen, U. 1998, A\&A, 329, 249

Kroupa, P. 2001, MNRAS, 322, 231

Krumholz, M. R., McKee, C. F., \& Klein, R. I. 2005, Nature, 438, 332

Lada, C. J., \& Lada, E. A. 2003, ARA\&A, 41, 57

Larson, R. B. 1985, MNRAS, 214, 379

Larson, R. B. 2005, MNRAS, 359, 211

Leung, C. M., \& Brown, R. L. 1977, ApJ, 214, L73

Lombardi, M., \& Bertin, G. 2001, A\&A, 375, 1091

Loren, R. B. 1989, ApJ, 338, 925

Loren, R. B., Wootten, A., \& Wilking, B. A. 1990, ApJ, 365, 269

Lovas, F. J. 1992, J. Phys. Chem. Ref. Data, 21, 181

Luhman, K. L., Rieke, G. H., Young, E. T., et al. 2000, ApJ, 540, 1016

Motte, F., \& André, P. 2001, in From Darkness to Light, ed. T. Montmerle, \& P. André, ASP Conf. Ser., 243, 301

Motte, F., André, P., \& Neri, R. 1998, A\&A, 336, 150 (MAN98)

Motte, F., André, P., Ward-Thompson, D., \& Bontemps, S. 2001, A\&A, 372, L41

Motte, F., Schilke, P., \& Lis, D. 2003, ApJ, 582, 277

Myers, P. C. 1998, ApJ, 496, L109

Myers, P. C. 1999, in The Physics and Chemistry of the Interstellar Medium, ed.

V. Ossenkopf, J. Stutzki, \& G. Winnewisser (Herdecke: GCA-Verlag), 227

Myers, P. C. 2000, ApJ, 530, L119

Myers, P. C., Evans, N. J. II, \& Ohashi, N. 2000, in Protostars and Planets IV, ed.

V. Mannings, A. P. Boss, \& S. S. Russell (Tucson: Univ. of Arizona Press), 217

Nakano, T. 1998, ApJ, 494, 587

Nutter, D., Ward-Thompson, D., \& André, P. 2006, MNRAS, 368, 1833

Padoan, P., \& Nordlund, A.. 2002, ApJ, 576, 870

Peretto, N., André, P., \& Belloche, A. 2006, A\&A, 445, 979

Peretto, N., Hennebelle, P., \& André, P. 2007, A\&A, 464, 983

Pickett, H. M., Poynter, R. L., Cohen, E. A., et al. 1998, J. Quant. Spectrosc. Rad. Transf., 60, 883

Price, N. M., \& Podsiadlowski, P. 1995, MNRAS, 273, 1041

Salpeter, E. E. 1955, ApJ, 121, 161

Shu, F. 1977, ApJ, 214, 488

Shu, F. H., Adams, F. C., \& Lizano, S. 1987, ARA\&A, 25, 23

Shu, F. H., Li, Z.-Y., \& Allen, A. 2004, ApJ, 601, 930

Stamatellos, D., \& Whitworth, A. P. 2003, A\&A, 407, 941

Stanke, T., Smith, M. D., Gredel, R., \& Khanzadyan, T. 2006, A\&A, 447, 609

Starck, J.-L., Murtagh, F., \& Bijaoui, A. 1998, Image processing and data analysis. The multiscale approach (Cambridge, UK: Cambridge University Press) Stutzki, J., \& Güsten, R. 1990, ApJ, 356, 513

Testi, L., \& Sargent, A. I. 1998, ApJ, 508, L91

Troland, T. H., Crutcher, R. M., Goodman, A. A., et al. 1996, ApJ, 471, 302

Walsh, A. J., Bourke, T. L., \& Myers, P. C. 2006, ApJ, 637, 860

Walsh, A. J., Myers, P. C., Di Francesco, J., et al. 2007, ApJ, 655, 958

Ward-Thompson, D., André, Ph., Crutcher, R., et al. 2007, Protostars and Planets V, ed. B. Reipurth, D. Jewitt, \& K. Keil (Tucson: University of Arizona Press), 33

Wilking, B. A., \& Lada, C. J. 1983, ApJ, 274, 698

Zeng, Q., Batrla, W., \& Wilson, T. L. 1984, A\&A, 141, 127 